Article

\title{
A Network Slicing Framework for UAV-Aided Vehicular Networks
}

\author{
Emmanouil Skondras ${ }^{1}$, Emmanouel T. Michailidis ${ }^{2} * \mathbb{D}$, Angelos Michalas ${ }^{3}$, Dimitrios J. Vergados ${ }^{4}$, \\ Nikolaos I. Miridakis ${ }^{5}$ and Dimitrios D. Vergados ${ }^{1}$
}

1 Department of Informatics, University of Piraeus, 80 Karaoli \& Dimitriou St., 18534 Piraeus, Greece; skondras@unipi.gr (E.S.); vergados@unipi.gr (D.D.V.)

2 Department of Electrical and Electronics Engineering, University of West Attica, Ancient Olive Grove Campus, 250 Thivon \& P. Ralli, Egaleo, 12244 Athens, Greece

3 Department of Electrical and Computer Engineering, University of Western Macedonia, Karamanli \& Ligeris, 50131 Kozani, Greece; amichalas@uowm.gr

4 Department of Informatics, University of Western Macedonia, Fourka Area, 52100 Kastoria, Greece; dvergados@uowm.gr

5 Department of Informatics and Computer Engineering, University of West Attica, Egaleo Park, Ag. Spyridonos Str., Egaleo, 12243 Athens, Greece; nikozm@uniwa.gr

* Correspondence: emichail@uniwa.gr

Citation: Skondras, E.; Michailidis, E.T.; Michalas, A.; Vergados, D.J.; Miridakis, N.I.; Vergados, D.D. A Network Slicing Framework for UAV-Aided Vehicular Networks. Drones 2021, 5, 70. https://doi.org/ $10.3390 /$ drones 5030070

Academic Editor: Abderrahmane Lakas

Received: 20 June 2021

Accepted: 23 July 2021

Published: 30 July 2021

Publisher's Note: MDPI stays neutral with regard to jurisdictional claims in published maps and institutional affiliations.

Copyright: (c) 2021 by the authors. Licensee MDPI, Basel, Switzerland. This article is an open access article distributed under the terms and conditions of the Creative Commons Attribution (CC BY) license (https:/ / creativecommons.org/licenses/by/ $4.0 /)$.
Abstract: In a fifth generation (5G) vehicular network architecture, several point of access (PoA) types, including both road side units (RSUs) and aerial relay nodes (ARNs), can be leveraged to undertake the service of an increasing number of vehicular users. In such an architecture, the application of efficient resource allocation schemes is indispensable. In this direction, this paper describes a network slicing scheme for $5 \mathrm{G}$ vehicular networks that aims to optimize the performance of modern network services. The proposed architecture consists of ground RSUs and unmanned aerial vehicles (UAVs) acting as ARNs enabling the communication between ground vehicular nodes and providing additional communication resources. Both RSUs and ARNs implement the LTE vehicle-to-everything (LTE-V2X) technology, while the position of each ARN is optimized by applying a fuzzy multi-attribute decision-making (fuzzy MADM) technique. With regard to the proposed network architecture, each RSU maintains a local virtual resource pool (LVRP) which contains local RBs (LRBs) and shared RBs (SRBs), while an SDN controller maintains a virtual resource pool (VRP), where the SRBs of the RSUs are stored. In addition, each ARN maintains its own resource blocks (RBs). For users connected to the RSUs, if the remaining RBs of the current RSU can satisfy the predefined threshold value, the LRBs of the RSU are allocated to user services. On the contrary, if the remaining RBs of the current RSU cannot satisfy the threshold, extra RBs from the VRP are allocated to user services. Similarly, for users connected to ARNs, the satisfaction grade of each user service is monitored considering both the QoS and the signal-to-noise plus interference (SINR) factors. If the satisfaction grade is higher than the predefined threshold value, the service requirements can be satisfied by the remaining RBs of the ARN. On the contrary, if the estimated satisfaction grade is lower than the predefined threshold value, the ARN borrows extra RBs from the LVRP of the corresponding RSU to achieve the required satisfaction grade. Performance evaluation shows that the suggested method optimizes the resource allocation and improves the performance of the offered services in terms of throughput, packet transfer delay, jitter and packet loss ratio, since the use of ARNs that obtain optimal positions improves the channel conditions observed from each vehicular user.

Keywords: 5G vehicular networks; unmanned aerial vehicles (UAVs); fuzzy multi-attribute decisionmaking (fuzzy MADM); LTE advance pro with FD-MIMO (LTE-A Pro FD-MIMO); LTE vehicle-toeverything (LTE-V2X); network slicing; computation offloading; software-defined networking (SDN) 


\section{Introduction}

In recent years, network slicing has rapidly evolved, providing novel techniques for performing resource allocation in fifth generation (5G) network infrastructures. One of the most important developments that network slicing has enabled is that of the virtualization of radio access network (RAN) resources [1]. As a result, virtual communication resources can be shared between many points of access (PoAs) by taking into consideration the current demands of user services. Thus, PoAs with saturated communication resources can borrow additional resources from their neighboring PoAs in order to satisfy the increased requirements of user services.

Several RAN technologies can be used to construct RAN infrastructures with both ground and aerial PoAs. Indicatively, in a 5G vehicular network, road side units (RSUs) can implement the long-term evolution of vehicle-to-everything (LTE-V2X) [2] technology, while unmanned aerial vehicles (UAVs) [3] can simultaneously act as aerial relay nodes (ARNs) [4] that provide additional communication resources for user services, as well as improve the channel conditions for users that receive decreased SINR from their RSU. Additionally, in a vehicular network environment, vehicles usually serve many passengers with multiple services each. Thus, the optimal allocation of the available communication resource, called resource blocks (RBs) [5] in the case of the LTE technology, is a critical challenge which must be addressed.

To satisfy the aforementioned requirement of the improved allocation of communication resources, this paper proposes a novel network scheme for performing network slicing on a $5 \mathrm{G}$ vehicular network. This scheme aims to improve the performance of vehicular services such as autonomous navigation (ANav), conversational voice (CVo), conversational video (CVi) and Web browsing (WB). Moreover, this scheme was deployed to a novel network architecture that consists of UAVs acting as ARNs and RSUs, providing RBs to vehicular users. Moreover, the position of each ARN is optimized by applying a fuzzy multi-attribute decision-making (fuzzy MADM) method which is implemented using interval valued icosagonal fuzzy numbers (IVIFNs) [6]. Regarding the design of the proposed scheme, each ARN maintains its own RBs, while each RSU maintains a local virtual resource pool, called LVRP, where both LRBs and SRBs exist. Furthermore, a software-defined networking (SDN) controller maintains a global virtual resource pool, called VRP, where the SRBs of the RSUs are stored. For users connected to either the ARNs or the RSUs, the satisfaction grade of their services is monitored considering both the quality of service (QoS) and the signal-to-noise plus interference (SINR) parameters. If the satisfaction grade is higher than the predefined threshold value, the service requirements can be satisfied from the remaining local RBs of the ARN or the RSU, respectively. On the contrary, if the satisfaction grade is lower than the predefined threshold, the ARN or the RSU borrows extra RBs from the LVRP or the VRP, respectively.

The proposed approach includes the following characteristics:

- A three-layer architecture is implemented for the optimal allocation of communication resources to user services.

- Both RSUs and ARNs are deployed to undertake the service of vehicular users.

- The satisfaction grade of user services, considered during the network slicing process, is estimated using the Mamdani fuzzy inference system (MFIS).

- Both the QoS and the SINR are considered for the estimation of the satisfaction grade of user services.

- A fuzzy MADM algorithm is used for the optimization of the position of each ARN.

- ARNs can commit additional communication resources from the LVRP of the corresponding RSUs to meet the requirements of the services of their users.

- An SDN controller maintains a VRP allowing the RSUs to commit additional communication resources to satisfy the strict requirements of users' services.

- Both the MFIS that estimates the satisfaction grade of user services and the fuzzy MADM algorithm that performs the optimization of the ARNs' positions are implemented using IVIFNs. 
The remainder of the paper is as follows: in Section 2, the current state of the art is revised, while Section 3 presents the proposed scheme for performing network slicing on $5 \mathrm{G}$ vehicular networks. Section 4 presents the simulation setup and Section 5 describes the evaluation results. Finally, Section 6 concludes the discussed work.

\section{State of the Art}

In recent years, various contributions in relevant technological areas have been reported upon which the proposed network slicing framework is based.

\subsection{Network Slicing in Vehicular Networks}

Several schemes for performing resource allocation on 5G network architectures, including 5G vehicular networks, have been proposed in the research literature. Indicatively, in order to optimize the performance of the 5G RAN environment, a scheme for the manipulation of network resources has been proposed based on the next-generation mobile network alliance (NGMN) $[7,8]$. In this scheme, each network slice employs multiple instances of logical subnetworks, while each subnetwork fulfills the requirements of specific user services. In addition to the NGMN's scheme, several network slicing schemes perform the resource allocation by applying either non-QoS-aware or QoS-aware strategies. Indicatively, in [9], three methods for performing network slicing by applying the proportional fairness (PF) [10] non-QoS-aware scheduler are proposed. The first method is called the static allocation (SA) method. It estimates the required number of RBs for each slice by taking into consideration the constraints of the slice's services. Subsequently, the PF algorithm is used for the allocation of the RBs that each slice has committed to vehicular users. Accordingly, the second method is called the allocation of ordered slices (AOS). Its functionality is similar to the one implemented by the SA method, while in this case, the priorities of the services that each slice serves are considered during the resource allocation. Finally, the third method is called impartial allocation (IA). This method is similar to AOS. The only difference is that, in this case, each slice commits RBs by not considering only the number of RBs that should be committed but also the channel quality that is observed in each RB. Thus, in this case, a service with higher priority allocates RBs with better channel quality.

Furthermore, the ultra-reliable low latency communication for autonomous vehicular networks (URLLC-AVN) QoS-aware algorithm described in [11] performs RAN virtualization to enhance the allocation of the communication resources in vehicular networks. In particular, in this case, each RSU maintains a set of RBs. The RBs of each RSU are divided into two subsets, which are referred to as local-RBs and as shared-RBs. The local-RBs of each specific RSU are only allocated to vehicular users that are connected to this RSU. On the other hand, the shared-RBs of each RSU are stored to a virtual resource pool. The queue delay that is observed for the services of each vehicular user is considered for the allocation of the shared RBs. Specifically, if the observed queue delay is lower than a delay threshold, then the local RBs are considered sufficient for satisfying the constraints of the services of the user. However, in cases where the observed queue delay is higher than the delay threshold, additional shared-RBs from the virtual resource pool are allocated to improve the performance of the services of the vehicular user.

The frame level scheduler (FLS) described in [12,13] is another QoS-aware algorithm used for resource allocation in $5 \mathrm{G}$ network infrastructures. This algorithm implements two levels, namely the upper and the lower level. The upper level estimates the quota of data that each real-time flow must transmit in order to satisfy its QoS constraints. Subsequently, the lower level initially allocates RBs to real-time flows using the PF algorithm, while the remaining RBs are allocated to non-real-time flows. Moreover, an improved version of the FLS algorithm, called FLS advanced (FLSA) is proposed in [14], where the performance of real-time services is further enhanced. Finally, another improved version of the algorithm, called FLS advanced with cross carrier (FLSA-CC) is described in [15] where the operating principles of cross carrier (CC) scheduling [16] are applied. 
In [17], the throughput enhanced scheduler (TES) is described. The TES algorithm defines two scheduling techniques. The first technique allocates resources to real-time flows by taking into consideration parameters such as the maximum acceptable delay of each service, the head of line (HOL) delay observed in each service queue, the SINR, the available throughput and the past average throughput observed for each service flow. Accordingly, the second technique is applied for the allocation of communication resources to non-real-time service. It must be noted that this technique does not take into consideration the target delay, but in this case, the maximum acceptable packet loss ratio is considered.

\subsection{The Emergence of Aerial Networks}

As the demand for comprehensive wireless communication services and ubiquitous access over large coverage areas has grown, the UAVs, widely known as drones, can strongly support the ground networks in propagation scenarios with obstacles and highly mobile and remote nodes [18-20]. In this direction, drone-to-everything (D2X) networks can complement the ground vehicle-to-everything (V2X) networks and significantly improve connectivity [21]. More specifically, the UAVs can fly at modest altitudes over connected vehicles and enable the establishment of an adaptable and reliable multi-hop communication backbone. A two-layer architecture for cooperative vehicular networking in challenging environments was presented in [22]. This architecture consists of multiple UAVs and ground components and enables relay-based, inter-UAV air-to-air (A2A), and air-to-ground (A2G) communications.

In order to facilitate the seamless integration of different heterogeneous networks consisting of ground and aerial entities, Cloud and Fog computing and networking have been previously suggested and network architectures with UAVs acting as providers of Fog computing services were proposed [23]. In addition, machine learning (ML) techniques have been exploited to improve various design and functional aspects of UAV-based communications [24]. In addition, the software-defined radio (SDR) and software-defined networking (SDN) can bring flexibility and cost-efficient deployment and runtime of customized networks [25]. A network architecture that integrates both ground and flying network nodes was proposed in [26] and intended to meet the requirements for extended radio coverage and increases sum-rate. This architecture takes advantage of moving radio access (RA) nodes, SDN, network functions virtualization (NFV), and energy-awareness for sustainable operation.

Previously, UAVs were also employed to construct air-ground integrated mobile edge networks $[27,28]$, since next-generation computation-intensive and delay-sensitive applications necessitate flexible network deployments and improved quality of the communication links. In order to obtain ultra-low latency in scenarios with large distances and during the processing of large data volumes, the use of computing resources at the edge of the network was also suggested in [29]. In particular, a flying ad hoc network (FANET) with multiple UAVs equipped with a mobile edge computing (MEC) server was envisioned as a key enabler for efficient computing. As the UAVs typically have certain constraints in terms of computing resources and battery capacity, a novel computation and offloading strategy relying on reinforcement learning (RL) was considered, where the onboard computing elements (CE) of the UAVs can be enabled or disabled according to the battery status using a system controller (SC). Based on this strategy, the computation task can be transferred to other UAVs in a cooperative manner, in order to satisfy a trade-off between power consumption, successful task processing, and delay.

As 5G network slicing aims to provide particular network resources to specific applications on demand, "AirSlice", a UAV-based network slicing framework, was presented in [30] as capable of supporting variable quality-of-service (QoS) requirements of UAVbased applications based on traffic classes. In addition, the feasibility of integrating UAVs as aerial nodes into 5G network slicing configurations for the enhanced reliability and robustness of control links was underlined in [31]. In addition, the efficacy of UAV-based 
network slicing along with mobile broadband services was experimentally validated in [32] for rail and vehicle scenarios using a $5 \mathrm{G}$ testbed. A service-oriented network slicing framework was proposed in [33] for an air-ground integrated vehicular network (AGIVEN) consisting of high-altitude platforms (HAPs), ground road side units (RSUs), and vehicles, in order to control multi-dimensional heterogeneous resources. In this regard, the AGIVEN was split into distinct virtual slices, each associated with particular applications and service requirements. In addition, a space-air-ground integrated vehicular network (SAGVN) was presented in [34] and the spectrum resources were dynamically sliced based on the demands of vehicular services. In [35], a 5G network slice framework was proposed that includes a FANET with UAVs capable of providing MEC services. This framework intended to provide improved reliability and decreased end-to-end latency between sources and actuators.

\subsection{Contribution}

This paper proposes a novel network framework that satisfies the requirements of $5 \mathrm{G}$ vehicular networking applications. More importantly, this framework extends the conventional network slicing concept and provides a flexible UAV-based network slicing that maintains wireless connections in challenging propagation areas, where vehicles struggle for connectivity.

The strong aspects of the URLLC-AVN [11], FLSA-CC [15] and TES [17] schemes, as well as of aerial networks, are combined to accomplish enhanced performance regarding the allocation of the RBs to vehicular users. In particular, the design of the URLLC-AVN was extended, resulting in a three-layer architecture according to the operating principles of the FLSA-CC algorithm, in a way similar to [36]. At the same time, the proposed architecture was further enhanced with the use of ARNs that provide additional communication resources and improved channel conditions. In this point, it should be noted that the geographical position of each ARN is optimized using a fuzzy MADM algorithm. Furthermore, for the allocation of RBs which is performed from the corresponding layers of the proposed architecture, the proposed network slicing scheme estimates the satisfaction grade of each user service using a Mamdani fuzzy inference system (FIS), and subsequently, an improved version of the TES, where the maximum acceptable packet loss ratio is considered both in real-time and non-real-time services, along with the other parameters that the TES algorithm considers in each case.

The evaluation of the satisfaction grade of user services using fuzzy logic can be considered a critical advantage of the proposed scheme against alternative solutions such as [30-35], since fuzzy logic enables the extraction of useful conclusions even if intermediate or approximate information is considered [37,38]. Furthermore, another advantage of the described methodology is the selection of the positions of the ARNs considering multiple criteria as input to a fuzzy MADM algorithm. As a result, each ARN obtains an optimal position enhancing the quality of the communication channel and thus, the performance of the entire $5 \mathrm{G}$ vehicular network architecture.

\section{The Proposed Network Slicing Scheme}

This paper leverages a three-layer design to optimize the allocation of the communication resources to user services. The network slicing scheme is deployed in a $5 \mathrm{G}$ network architecture, where each network component implements specific layers of the implemented layered stack. In the following subsections, both the design of the proposed scheme and the underlying network architecture are described.

\subsection{The Layered Design of the Proposed Scheme}

The proposed slicing scheme improves the three-layer design of the FLSA-CC [15] scheduler. In particular, as presented in Figure 1, two service groups are defined, namely the guaranteed bit rate (GBR) and the non-guaranteed bit rate (non-GBR) containing best effort services. Initially, the upper layer categorizes the GBR service flow into two 
subcategories. The first subcategory called delay critical GBR (DC-GBR) includes services where the observed delay is a very critical factor that should be satisfied. Indicatively, an autonomous navigation service flow with maximum acceptable delay up to $5 \mathrm{~ms}$ [39] is considered as a DC-GBR service. Accordingly, the second subcategory called non-delay critical GBR (DC-GBR) includes real-time services with increased tolerance to the delay factor (e.g., conversational video services). Indicatively, a conversational video service flow with maximum acceptable delay up to $150 \mathrm{~ms}$ [39] is considered as a nDC-GBR service. Subsequently, the upper layer evaluates the amount of RBs that should be committed for each DC-GBR and nDC-GBR service to satisfy its QoS requirements. Then, the middle layer allocates RBs obtained from the upper layer to both DC-GBR and nDC-GBR services. Finally, the lower layer allocates the remaining RBs to non-GBR services. The functionalities of each layer are described in the following subsections.

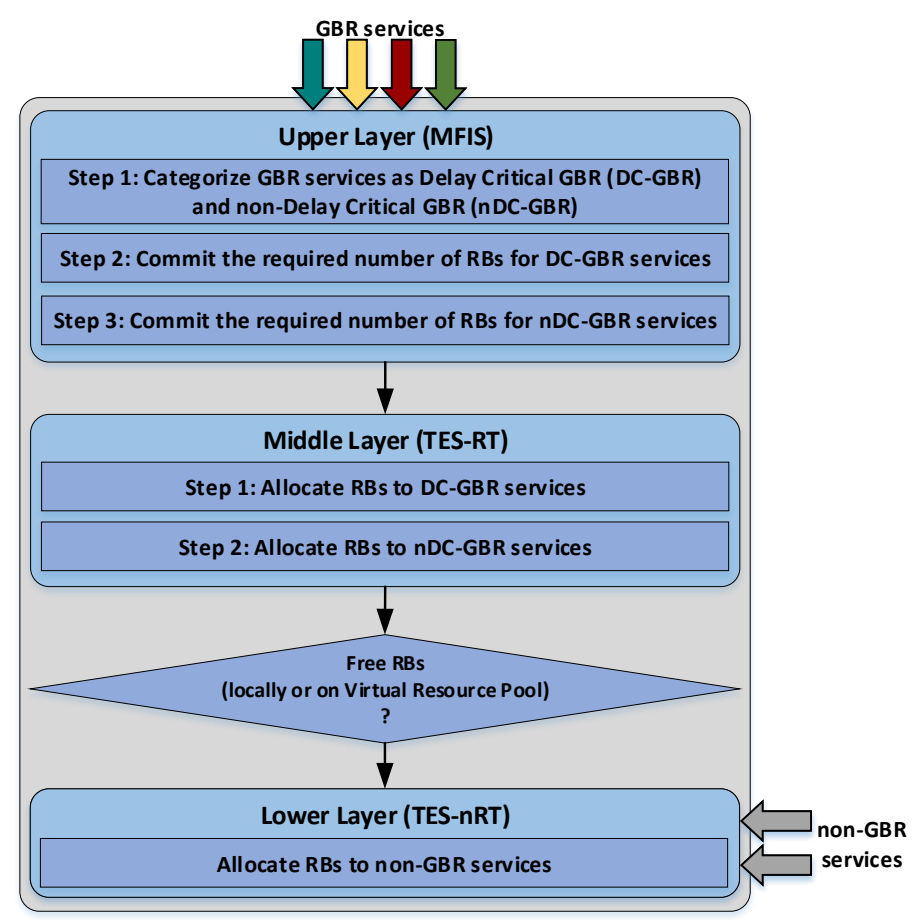

Figure 1. The layer stack of the proposed scheme.

\subsubsection{The Upper Layer of the Network Slicing Scheme}

A set $M_{u}\left(t_{n}\right)$ of GBR services per user $u$ requires communication resources to satisfy particular constraints during the $t_{n}$ TTI. Initially, the upper layer creates two subsets of services, namely the $M_{u, D C-G B R}\left(t_{n}\right)$ and the $M_{u, n D C-G B R}\left(t_{n}\right)$ consisting of the DC-GBR and the nDC-GBR services of the user, respectively. Subsequently, for each $m_{u, D C-G B R}\left(t_{n}\right) \in M_{u, D C-G B R}\left(t_{n}\right)$ service, the $S_{m, u}^{\text {estimated }}\left(t_{n}\right)$ indicator is defined. It determines the estimated satisfaction grade of user service $m_{u, D C-G B R}$ during the upcoming $t_{n}$ TTI.

The Mamdani fuzzy inference system (MFIS) system [40] is applied for the estimation of the $S_{m, u}^{\text {estimated }}\left(t_{n}\right)$. In this cases, both the $S I N R_{m, u}^{\text {estimated }}$ and the $Q_{m, u}^{\text {estimated }}$ parameters are considered inputs, while they are expressed as interval valued icosagonal fuzzy numbers (IVIFNs) [6]. The linguistic terms and the corresponding IVIFNs used for the representation of the $S_{m, u}^{\text {estimated }}$, the $S I N R_{m, u}^{\text {estimated }}$ and the $Q_{m, u}^{\text {estimated }}$ values are presented in Table 1 . In addition, Table 2 presents the fuzzy rules used for the estimation of the $S_{m, u}^{\text {estimated }}$ parameter.

In particular, the $S I N R_{m, u}^{\text {estimated }}$ parameter represents the SINR observed from the user $u$. Furthermore, the $Q_{m, u}^{\text {estimated }}\left(t_{n}\right)$ parameter represents the estimated quality of the $m_{u, D C-G B R}$ user's service regarding the available RBs in the $t_{n}$ TTI. $Q_{m, u}^{\text {estimated }}\left(t_{n}\right)$ is calculated using Formula (1) which applies the multiplicative exponent weighting 
(MEW) method described in [41]. In this formula, the $t h_{m, u}^{\text {estimated }}\left(t_{n}\right)$, the $d_{m, u}^{\text {estimated }}\left(t_{n}\right)$, the $j_{m, u}^{\text {estimated }}\left(t_{n}\right)$ and the $p l_{m, u}^{\text {estimated }}\left(t_{n}\right)$ parameters represent the normalized values of the throughput, the packet transfer delay, the jitter and the packet loss ratio parameters, respectively. In addition, the $w_{t h}, w_{d}, w_{j}$ and $w_{p l}$ represent the weights of the considered parameters. In this work, the aforementioned weights are calculated using the fuzzy analytic network process (FANP) method [40] which is implemented using IVPFNs:

$$
Q_{m, u}^{\text {estimated }}\left(t_{n}\right)=\left[\text { th }_{m, u}^{\text {estimated }}\left(t_{n}\right)\right]^{w_{t h}} \cdot\left[d_{m, u}^{\text {estimated }}\left(t_{n}\right)\right]^{w_{d}} \cdot\left[j_{m, u}^{\text {estimated }}\left(t_{n}\right)\right]^{w_{j}} \cdot\left[p_{m, u}^{\text {estimated }}\left(t_{n}\right)\right]^{w_{p l}}
$$

Furthermore, it should be noted that the value $t h_{m, u}^{\text {estimated }}\left(t_{n}\right)$ is calculated using Formula (2), where $\mu(t)$ is the estimated throughput per RB and $r^{\text {available }}$ represents the number of RBs that are available for allocation:

$$
t h_{m, u}^{\text {estimated }}\left(t_{n}\right)=\frac{\left(\mu\left(t_{n}\right) \cdot r^{\text {available }}\right)-t h^{\min }}{t h^{\max }-t h^{\min }}
$$

Moreover, the values of the $d_{m, u}^{\text {estimated }}\left(t_{n}\right), j_{m, u}^{\text {estimated }}\left(t_{n}\right)$ and $p l_{m, u}^{\text {estimated }}\left(t_{n}\right)$ parameters are calculated using Formulas (3)-(5), respectively. The $p_{m, u}^{\text {average }}$ parameter represents the average packet size, the $d_{m, u}^{\text {pastaverage }}$ is the past average packet transfer delay and the $t h_{m, u}^{\text {required }}$ is the required throughput of the $m^{\text {th }}$ service of user $u$ :

$$
\begin{gathered}
d_{m, u}^{\text {stimated }}\left(t_{n}\right)=1-\left[\frac{\left(\frac{p_{m, u}^{\text {average }}}{\text { th }_{m, u}^{\text {stimated }}\left(t_{n}\right)}\right)-d^{\text {min }}}{d^{\text {max }}-d^{\text {min }}}\right] \\
j_{m, u}^{\text {estimated }}\left(t_{n}\right)=1-\left[\frac{\left|d_{m, u}^{\text {estimated }}\left(t_{n}\right)-d_{m, u}^{\text {past_average }}\right|-j^{\text {min }}}{j^{\text {max }}-j^{\text {min }}}\right] \\
p l_{m, u}^{\text {estimated }}\left(t_{n}\right)=1-\left[\frac{\left(\frac{t_{m, u}^{\text {required }}\left(t_{n}\right)-t h_{m, u}^{\text {estimated }}\left(t_{n}\right)}{t h_{m, u}^{\text {required }}\left(t_{n}\right)}\right)-p l^{\text {min }}}{p l^{\text {max }}-p l^{\text {min }}}\right]
\end{gathered}
$$

The minimum acceptable satisfaction grades $S_{m, u}^{\text {threshold }}$ per user and service are obtained using the $S I N R_{m, u}^{\text {threshold }}$ and the $Q_{m, u}^{\text {threshold }}$ threshold values defined in [39]. Subsequently, the aforementioned procedure is also performed for each $m_{u, n D C-G B R}\left(t_{n}\right) \in$ $M_{u, n D C-G B R}\left(t_{n}\right)$ service.

The Mamdani Satisfaction Chart

During the instantiation of the system architecture, a Mamdani satisfaction chart, which contains the $S_{m, u}^{\text {estimated }}$ values obtained for each possible $S I N R_{m, u}^{\text {estimated }}$ and $Q_{m, u}^{\text {estimated }}$ combination, is created. Specifically, each satisfaction indicator $S_{m, u}^{\text {estimated }}$ of Figure 2 is obtained using the MFIS method, considering the $S I N R_{m, u}^{\text {estimated }}$ and the $Q_{m, u}^{\text {estimated }}$ as input parameters. Both $S I N R_{m, u}^{\text {estimated }}$ and $Q_{m, u}^{e s t i m a t e d}$ are normalized in order to have values within the range of $[0,1]$. In addition, the $M F_{S I N R}, M F_{Q}, M F_{S}$ membership functions (MFs) are defined using the equalized universe method (EUM) method [40], indicating the linguistic terms and the corresponding IVPFNs for the fuzzy representation of the SINR $R_{m, u}^{\text {estimated }}$, $Q_{m, u}^{e s t i m a t e d}$ and $S_{m, u}^{e s t i m a t e d}$ factor, respectively. Thus, for each crisp value, two membership degrees are determined in the corresponding $\mathrm{MF}$, one for the upper octagon and one for 
the lower octagon. Table 1 represents the linguistic terms and the corresponding IVPFNs of $M F_{S I N R}, M F_{Q}$ and $M F_{S}$ membership functions, which are equally distributed inside the domain $\left[U_{\min }, U_{\max }\right]=[0,1]$, as described in [40]. Furthermore, Table 2 presents the considered fuzzy rule base which is used from the MFIS for producing the satisfaction chart.

Table 1. The linguistic terms used for the representation of $M F_{S I N R}, M F_{Q}$ and $M F_{S}$ membership functions and the corresponding interval-valued icosagonal fuzzy numbers .

\begin{tabular}{|c|c|c|c|}
\hline $\begin{array}{c}\text { Linguistic Terms for } \\
\text { the } M F_{S I N R} \\
\text { Membership Functions }\end{array}$ & $\begin{array}{l}\text { Linguistic Terms for } \\
\text { the } M F_{Q} \text { Membership } \\
\text { Functions }\end{array}$ & $\begin{array}{l}\text { Linguistic Terms for the } M F_{S} \\
\text { Membership Functions }\end{array}$ & $\begin{array}{l}\text { Interval-Valued Icosagonal Fuzzy } \\
\text { Number }\end{array}$ \\
\hline Absolutely Bad (AB) & Absolutely Poor (AP) & $\begin{array}{l}\text { Absolutely Unsatisfactory } \\
\text { (AU) }\end{array}$ & $\begin{array}{l}{[(0,0,0,0,0,0,0,0,0,0,0.008,0.023,} \\
0.038,0.053,0.068,0.083,0.098,0.113, \\
0.128,0.143,1,0.20,0.40,0.60,0.80,0.80, \\
0.60,0.40,0.20),(0,0,0,0,0,0,0,0,0,0, \\
0.006,0.018,0.030,0.042,0.054,0.066, \\
0.078,0.090,0.102,0.114,0.8,0.08,0.26, \\
0.44,0.62,0.62,0.44,0.26,0.08)]\end{array}$ \\
\hline Too Bad (TB) & Very Poor (VP) & Very Unsatisfactory (VU) & $\begin{array}{l}{[(0,0,0,0,0.028,0.043,0.058,0.074,0.089,} \\
0.104,0.119,0.134,0.149,0.164,0.179, \\
0.194,0.209,0.224,0.239,0.254,1,0.20, \\
0.40,0.60,0.80,0.80,0.60,0.40,0.20),(0, \\
0.009,0.021,0.033,0.045,0.057,0.069, \\
0.081,0.093,0.105,0.117,0.129,0.141, \\
0.153,0.165,0.177,0.189,0.201,0.213, \\
0.225,0.8,0.08,0.26,0.44,0.62,0.62,0.44, \\
0.26,0.08)]\end{array}$ \\
\hline $\operatorname{Bad}(\mathrm{B})$ & Poor $(\mathrm{P})$ & Unsatisfactory (U) & $\begin{array}{l}{[(0.079,0.094,0.109,0.124,0.140,0.155,} \\
0.170,0.185,0.200,0.215,0.230,0.245, \\
0.260,0.275,0.290,0.305,0.320,0.335, \\
0.350,0.365,1,0.20,0.40,0.60,0.80,0.80, \\
0.60,0.40,0.20),(0.108,0.120,0.132,0.144, \\
0.156,0.168,0.180,0.192,0.204,0.216, \\
0.228,0.240,0.252,0.264,0.276,0.288, \\
0.300,0.312,0.324,0.337,0.8,0.08,0.26, \\
0.44,0.62,0.62,0.44,0.26,0.08)]\end{array}$ \\
\hline Less than Enough (LE) & Less than Medium (LM) & Less than Acceptable (LA) & $\begin{array}{l}{[(0.190,0.206,0.221,0.236,0.251,0.266,} \\
0.281,0.296,0.311,0.326,0.341,0.356, \\
0.371,0.386,0.401,0.416,0.431,0.446, \\
0.461,0.476,1,0.20,0.40,0.60,0.80,0.80, \\
0.60,0.40,0.20),(0.219,0.231,0.243,0.255, \\
0.267,0.279,0.291,0.303,0.315,0.327, \\
0.339,0.351,0.363,0.375,0.387,0.399, \\
0.412,0.424,0.436,0.448,0.8,0.08,0.26, \\
0.44,0.62,0.62,0.44,0.26,0.08)]\end{array}$ \\
\hline Enough (EN) & Medium (M) & Acceptable (A) & $\begin{array}{l}{[(0.302,0.317,0.332,0.347,0.362,0.377,} \\
0.392,0.407,0.422,0.437,0.452,0.467, \\
0.482,0.497,0.512,0.527,0.542,0.557, \\
0.572,0.587,1,0.20,0.40,0.60,0.80,0.80, \\
0.60,0.40,0.20),(0.330,0.342,0.354,0.366, \\
0.378,0.390,0.402,0.414,0.426,0.438, \\
0.450,0.462,0.475,0.487,0.499,0.511, \\
0.523,0.535,0.547,0.559,0.8,0.08,0.26, \\
0.44,0.62,0.62,0.44,0.26,0.08)]\end{array}$ \\
\hline
\end{tabular}


Table 1. Cont.

\begin{tabular}{|c|c|c|c|}
\hline $\begin{array}{c}\text { Linguistic Terms for } \\
\text { the } M F_{S I N R} \\
\text { Membership Functions }\end{array}$ & $\begin{array}{l}\text { Linguistic Terms for } \\
\text { the } M F_{Q} \text { Membership } \\
\text { Functions }\end{array}$ & $\begin{array}{l}\text { Linguistic Terms for the } M F_{S} \\
\text { Membership Functions }\end{array}$ & $\begin{array}{l}\text { Interval-Valued Icosagonal Fuzzy } \\
\text { Number }\end{array}$ \\
\hline More than Enough (ME) & $\begin{array}{l}\text { More than Medium } \\
\text { (MM) }\end{array}$ & More than Acceptable (MA) & $\begin{array}{l}{[(0.413,0.428,0.443,0.458,0.473,0.488,} \\
0.503,0.518,0.533,0.548,0.563,0.578, \\
0.593,0.608,0.623,0.638,0.653,0.668, \\
0.683,0.698,1,0.20,0.40,0.60,0.80,0.80, \\
0.60,0.40,0.20),(0.441,0.453,0.465,0.477, \\
0.489,0.501,0.513,0.525,0.538,0.550, \\
0.562,0.574,0.586,0.598,0.610,0.622, \\
0.634,0.646,0.6580 .670,0.8,0.08,0.26, \\
0.44,0.62,0.62,0.44,0.26,0.08)]\end{array}$ \\
\hline Less than Excellent (LE) & Less than Good (LG) & Slightly Satisfactory (SS) & $\begin{array}{l}{[(0.524,0.539,0.554,0.569,0.584,0.599,} \\
0.614,0.629,0.644,0.659,0.674,0.689, \\
0.704,0.719,0.734,0.749,0.764,0.779, \\
0.794,0.810,1,0.20,0.40,0.60,0.80,0.80, \\
0.60,0.40,0.20),(0.552,0.564,0.576,0.588, \\
0.601,0.613,0.625,0.637,0.649,0.661, \\
0.673,0.685,0.697,0.709,0.721,0.733, \\
0.745,0.757,0.769,0.781,0.8,0.08,0.26, \\
0.44,0.62,0.62,0.44,0.26,0.08)]\end{array}$ \\
\hline Almost Excellent (AE) & Good (G) & Satisfactory (S) & $\begin{array}{l}{[(0.635,0.650,0.665,0.680,0.695,0.710,} \\
0.725,0.740,0.755,0.770,0.785,0.800, \\
0.815,0.830,0.845,0.860,0.876,0.891, \\
0.906,0.921,1,0.20,0.40,0.60,0.80,0.80, \\
0.60,0.40,0.20),(0.663,0.676,0.688,0.700, \\
0.712,0.724,0.736,0.748,0.760,0.772, \\
0.784,0.796,0.808,0.820,0.832,0.844, \\
0.856,0.868,0.880,0.892,0.8,0.08,0.26, \\
0.44,0.62,0.62,0.44,0.26,0.08)]\end{array}$ \\
\hline Excellent (EX) & More than Good (MG) & Very Satisfactory (VS) & $\begin{array}{l}{[(0.746,0.761,0.776,0.791,0.806,0.821,} \\
0.836,0.851,0.866,0.881,0.896,0.911, \\
0.926,0.942,0.957,0.972,0.987,1,1,1,1, \\
0.20,0.40,0.60,0.80,0.80,0.60,0.40,0.20), \\
(0.775,0.787,0.799,0.811,0.823,0.835, \\
0.847,0.859,0.871,0.883,0.895,0.907, \\
0.919,0.931,0.943,0.955,0.967,0.979, \\
0.991,1,0.8,0.08,0.26,0.44,0.62,0.62, \\
0.44,0.26,0.08)]\end{array}$ \\
\hline $\begin{array}{l}\text { Absolutely Excellent } \\
\text { (AX) }\end{array}$ & Absolutely Good (AG) & Absolutely Satisfactory (AS) & $\begin{array}{l}{[(0.857,0.872,0.887,0.902,0.917,0.932,} \\
0.947,0.962,0.977,0.992,1,1,1,1,1,1,1, \\
1,1,1,1,0.20,0.40,0.60,0.80,0.80,0.60, \\
0.40,0.20),(0.886,0.898,0.910,0.922, \\
0.934,0.946,0.958,0.970,0.982,0.994,1,1, \\
1,1,1,1,1,1,1,1,0.8,0.08,0.26,0.44,0.62, \\
0.62,0.44,0.26,0.08)]\end{array}$ \\
\hline
\end{tabular}




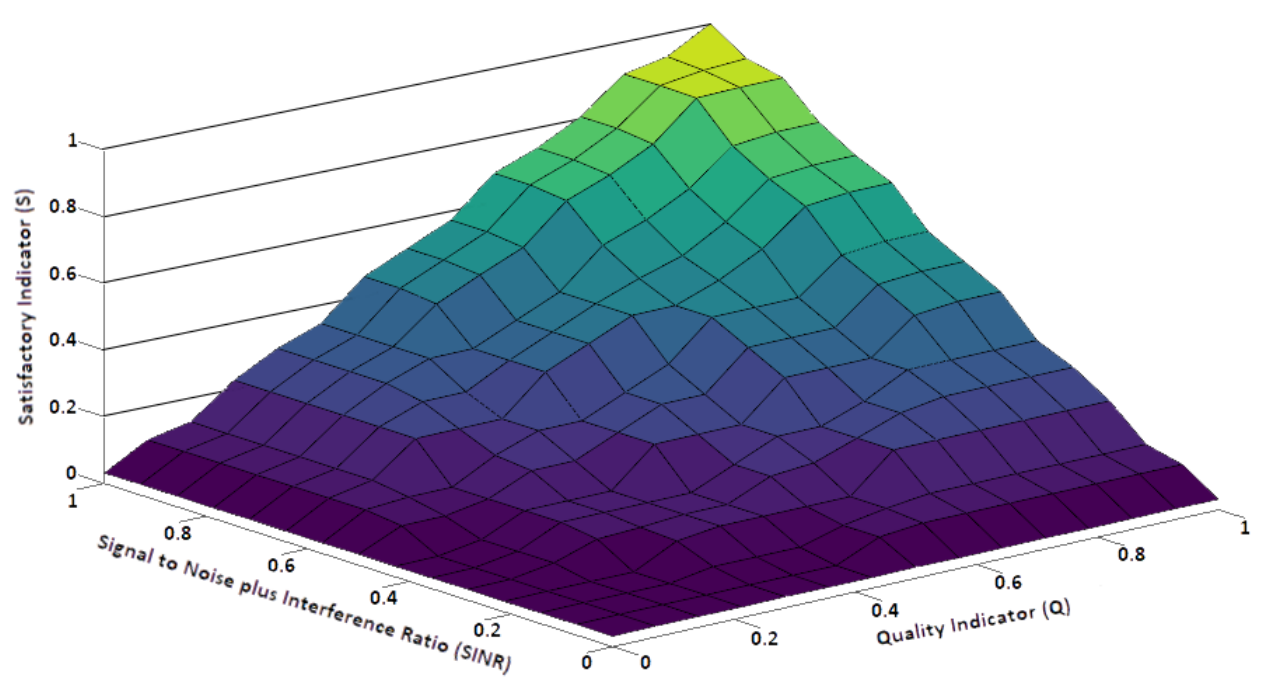

Figure 2. The $S$ values range as obtained using the Mamdani fuzzy Inference System.

Indicatively, when the $S I N R_{m, u}^{\text {estimated }}$ and $Q_{m, u}^{\text {estimated }}$ values are too low, the produced $S_{m, u}^{e s t i m a t e d}$ value is too low as well. On the contrary, when the $\operatorname{SIN} R_{m, u}^{\text {estimated }}$ and $Q_{m, u}^{\text {estimated }}$ values are close to 1 , the produced $S_{m, u}^{e s t i m a t e d}$ value is also high, indicating that the user is fully satisfied. Furthermore, when only one of the $S I N R_{m, u}^{\text {estimated }}$ or the $Q_{m, u}^{\text {estimated }}$ values is close to 0 , the user satisfaction is in low levels.

At this point, it has to be noted that since the user's satisfaction is obtained at the Fog by looking up the performance on the satisfaction indicators' chart, the overhead introduced is minimal. In addition, the method does not impose any significant overhead at the user equipment due to the monitoring of the $S I N R_{m, u}$ and $Q_{m, u}$ parameters.

\subsubsection{The Middle Layer of the Network Slicing Scheme}

In each TTI, the middle layer allocates to each GBR service the number of RBs estimated from the upper layer. The allocation is performed by applying an improved version of the scheduling metric that the throughput enhanced scheduler (TES) [17] algorithm defines for real-time services, where the maximum acceptable packet loss ratio is considered along with the other parameters that consider the aforementioned metric. Specifically, this metric is calculated using Formula (6), where $\tau_{i}$ stands for the maximum acceptable delay, $D H O L_{i}$ represents the head of line delay, $b$ is a constant, $\operatorname{SINR}_{i}$ represents the signal-to-interference plus noise ratio, $r_{i}$ is the available throughput and $R_{i}(t)$ is the past average throughput for the $i$ th flow. The $\alpha_{i}$ value is calculated using Formula (7) where $\delta_{i}$ represents the maximum acceptable packet loss ratio:

$$
\begin{gathered}
m_{i, k}^{T E S-R T_{\text {improved }}}=\alpha_{i} \cdot \exp \left(-\frac{\tau_{i}-D H O L_{i}}{\tau_{i}}\right) \cdot \log \left(b \cdot S I N R_{i}\right) \cdot \frac{r_{i}}{R_{i}(t)} \\
\alpha_{i}=-\frac{\log \delta_{i}}{\tau_{i}}
\end{gathered}
$$


Table 2. The fuzzy rule (or knowledge) base.

\begin{tabular}{|c|c|c|c|c|}
\hline Rule & $M F_{S I N R}$ & Operator & $M F_{Q}$ & $M F_{S}$ \\
\hline 1 & $\mathrm{AB}$ & and & $\mathrm{AP}$ & $\mathrm{AU}$ \\
\hline 2 & $\mathrm{AB}$ & and & VP & AU \\
\hline 3 & $\mathrm{AB}$ & and & $\mathrm{P}$ & $\mathrm{AU}$ \\
\hline 4 & $\mathrm{AB}$ & and & $\mathrm{LM}$ & AU \\
\hline 5 & $\mathrm{AB}$ & and & M & $\mathrm{AU}$ \\
\hline 6 & $\mathrm{AB}$ & and & $\mathrm{MM}$ & $\mathrm{AU}$ \\
\hline 7 & $\mathrm{AB}$ & and & LG & AU \\
\hline 8 & $\mathrm{AB}$ & and & G & $\mathrm{AU}$ \\
\hline 9 & $\mathrm{AB}$ & and & MG & AU \\
\hline 10 & $\mathrm{AB}$ & and & AG & $\mathrm{AU}$ \\
\hline 11 & TB & and & $\mathrm{AP}$ & AU \\
\hline 12 & $\mathrm{~TB}$ & and & $\mathrm{VP}$ & AU \\
\hline 13 & TB & and & $\mathrm{P}$ & $\mathrm{AU}$ \\
\hline 14 & $\mathrm{~TB}$ & and & $\mathrm{LM}$ & $\mathrm{AU}$ \\
\hline 15 & $\mathrm{~TB}$ & and & $\mathrm{M}$ & $\mathrm{AU}$ \\
\hline 16 & $\mathrm{~TB}$ & and & $\mathrm{MM}$ & VU \\
\hline 17 & $\mathrm{~TB}$ & and & LG & VU \\
\hline 18 & $\mathrm{~TB}$ & and & $\mathrm{G}$ & VU \\
\hline 19 & TB & and & MG & VU \\
\hline 20 & $\mathrm{~TB}$ & and & $\mathrm{AG}$ & VU \\
\hline 21 & B & and & $\mathrm{AP}$ & $\mathrm{AU}$ \\
\hline 22 & B & and & $\mathrm{VP}$ & $\mathrm{AU}$ \\
\hline 23 & B & and & $\mathrm{P}$ & $\mathrm{AU}$ \\
\hline 24 & B & and & $\mathrm{LM}$ & VU \\
\hline 25 & B & and & $\mathrm{M}$ & VU \\
\hline 26 & B & and & $\mathrm{MM}$ & VU \\
\hline 27 & B & and & LG & $\mathrm{U}$ \\
\hline 28 & B & and & G & $\mathrm{U}$ \\
\hline 29 & B & and & MG & $\mathrm{U}$ \\
\hline 30 & B & and & AG & $\mathrm{U}$ \\
\hline 31 & LE & and & $\mathrm{AP}$ & $\mathrm{AU}$ \\
\hline 32 & $\mathrm{LE}$ & and & $\mathrm{VP}$ & $\mathrm{AU}$ \\
\hline 33 & LE & and & $\mathrm{P}$ & VU \\
\hline 34 & LE & and & $\mathrm{LM}$ & VU \\
\hline 35 & LE & and & $\mathrm{M}$ & VU \\
\hline 36 & LE & and & $\mathrm{MM}$ & $\mathrm{U}$ \\
\hline 37 & LE & and & LG & $\mathrm{U}$ \\
\hline 38 & LE & and & G & LA \\
\hline 39 & LE & and & MG & LA \\
\hline 40 & LE & and & AG & LA \\
\hline 41 & EN & and & $\mathrm{AP}$ & $\mathrm{AU}$ \\
\hline 42 & EN & and & $\mathrm{VP}$ & $\mathrm{AU}$ \\
\hline 43 & EN & and & $\mathrm{P}$ & VU \\
\hline 44 & EN & and & $\mathrm{LM}$ & VU \\
\hline 45 & EN & and & $\mathrm{M}$ & $\mathrm{U}$ \\
\hline 46 & EN & and & $\mathrm{MM}$ & $\mathrm{U}$ \\
\hline 47 & EN & and & LG & LA \\
\hline 48 & EN & and & $\mathrm{G}$ & LA \\
\hline 49 & EN & and & MG & A \\
\hline 50 & EN & and & $\mathrm{AG}$ & A \\
\hline 51 & $\mathrm{ME}$ & and & $\mathrm{AP}$ & $\mathrm{AU}$ \\
\hline 52 & ME & and & $\mathrm{VP}$ & VU \\
\hline 53 & $\mathrm{ME}$ & and & $\mathrm{P}$ & VU \\
\hline 54 & $\mathrm{ME}$ & and & $\mathrm{LM}$ & $\mathrm{U}$ \\
\hline
\end{tabular}


Table 2. Cont.

\begin{tabular}{|c|c|c|c|c|}
\hline Rule & $M F_{S I N R}$ & Operator & $M F_{Q}$ & $M F_{S}$ \\
\hline 55 & $\mathrm{ME}$ & and & $\mathrm{M}$ & $\mathrm{U}$ \\
\hline 56 & $\mathrm{ME}$ & and & $\mathrm{MM}$ & LA \\
\hline 57 & $\mathrm{ME}$ & and & LG & A \\
\hline 58 & $\mathrm{ME}$ & and & G & A \\
\hline 59 & $\mathrm{ME}$ & and & MG & MA \\
\hline 60 & $\mathrm{ME}$ & and & AG & MA \\
\hline 61 & LE & and & $\mathrm{AP}$ & AU \\
\hline 62 & $\mathrm{LE}$ & and & VP & VU \\
\hline 63 & LE & and & $\mathrm{P}$ & $\mathrm{U}$ \\
\hline 64 & LE & and & LM & $\mathrm{U}$ \\
\hline 65 & LE & and & M & LA \\
\hline 66 & LE & and & MM & $\mathrm{A}$ \\
\hline 67 & LE & and & LG & A \\
\hline 68 & LE & and & $G$ & MA \\
\hline 69 & LE & and & MG & SS \\
\hline 70 & $\mathrm{LE}$ & and & $\mathrm{AG}$ & SS \\
\hline 71 & $\mathrm{AE}$ & and & $\mathrm{AP}$ & $\mathrm{AU}$ \\
\hline 72 & $\mathrm{AE}$ & and & VP & VU \\
\hline 73 & $\mathrm{AE}$ & and & $\mathrm{P}$ & $\mathrm{U}$ \\
\hline 74 & $\mathrm{AE}$ & and & LM & LA \\
\hline 75 & $\mathrm{AE}$ & and & $\mathrm{M}$ & LA \\
\hline 76 & $\mathrm{AE}$ & and & MM & A \\
\hline 77 & $\mathrm{AE}$ & and & LG & MA \\
\hline 78 & $\mathrm{AE}$ & and & G & SS \\
\hline 79 & $\mathrm{AE}$ & and & MG & $S$ \\
\hline 80 & $\mathrm{AE}$ & and & $A G$ & S \\
\hline 81 & EX & and & $\mathrm{AP}$ & $\mathrm{AU}$ \\
\hline 82 & EX & and & VP & VU \\
\hline 83 & EX & and & $\mathrm{P}$ & $\mathrm{U}$ \\
\hline 84 & EX & and & LM & LA \\
\hline 85 & EX & and & $\mathrm{M}$ & $\mathrm{A}$ \\
\hline 86 & EX & and & MM & MA \\
\hline 87 & EX & and & LG & SS \\
\hline 88 & EX & and & G & $S$ \\
\hline 89 & EX & and & MG & VS \\
\hline 90 & EX & and & AG & VS \\
\hline 91 & $A X$ & and & $\mathrm{AP}$ & $\mathrm{AU}$ \\
\hline 92 & $A X$ & and & VP & VU \\
\hline 93 & $\mathrm{AX}$ & and & $\mathrm{P}$ & $\mathrm{U}$ \\
\hline 94 & $A X$ & and & LM & LA \\
\hline 95 & $A X$ & and & $\mathrm{M}$ & $\mathrm{A}$ \\
\hline 96 & $A X$ & and & $\mathrm{MM}$ & MA \\
\hline 97 & $A X$ & and & LG & SS \\
\hline 98 & $A X$ & and & $\mathrm{G}$ & $S$ \\
\hline 99 & $A X$ & and & MG & VS \\
\hline 100 & $A X$ & and & $\mathrm{AG}$ & AS \\
\hline
\end{tabular}

3.1.3. The Lower Layer of the Network Slicing Scheme

The lower layer allocates available RBs to best effort flows using the scheduling metric that the TES [17] algorithm defines for non-real-time services. In particular, the metric is estimated using Formula (8):

$$
m_{i, k}^{T E S-n R T}=\alpha_{i} \cdot \mathrm{DHOL}_{i} \cdot \log \left(b \cdot \operatorname{SINR}_{i}\right) \cdot \frac{r_{i}}{R_{i}(t)}
$$




\subsection{The Proposed Network Architecture}

The network architecture (Figure 3) consists ARNs and RSUs that facilitate the provision of network access to vehicular users. In particular, the coverage area of the access network is divided into $K$ cells while each cell is served from an ARN and an RSU.

Each $k \in K$ ARN $\left(A R N_{k}\right)$ has a local monitoring module (LMM), a local allocation module (LAM) and a set of resource blocks (RBs). A variable $R B_{A R N_{k}}^{r e m}\left(t_{n}\right)$ indicates the remaining RBs of the $k$ th ARN during the $t_{n}$ transmission time interval (TTI). Similarly, each $k \in K$ RSU $\left(R S U_{k}\right)$ implements its local monitoring module (LMM), its local allocation module (LAM) and a local virtual resource pool (LVRP) with a set of resource blocks (RBs). A variable $R B_{R S U_{k}}^{r e m}\left(t_{n}\right)$ indicates the remaining RBs of the $k$ th RSU during the $t_{n}$ TTI. In addition, the RBs of the LVRP are organized into two subsets, which are called local RBs (LRBs) and shared RBs (SRBs).

An SDN controller maintains a virtual resource pool (VRP) where the SRBs of the RSUs are stored, in a way similar to [36,42]. In addition, the SDN controller includes an allocation module (AM) and a MANO entity which orchestrates the entire network slicing procedure whilst maintaining the necessary frequency reuse factor. At this point, it has to be noted that in order to ensure the scalability of the proposed scheme, multiple SDN controllers can be used. In this case, each SDN controller will maintain a VRP for a specific geographical area in a way similar to that described in [36]. Thus, as the system expands, additional SDN controllers can be used to control the VRPs of each subarea of the entire system coverage.

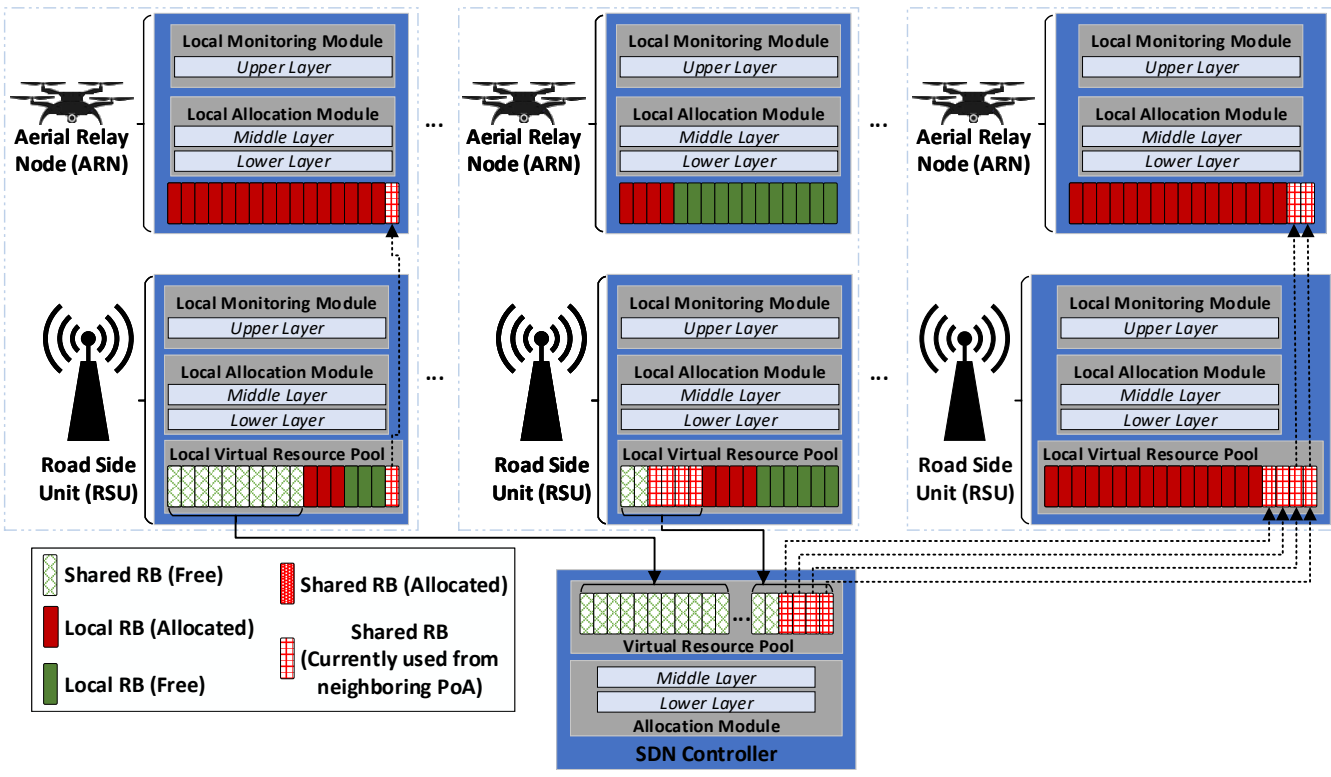

Figure 3. The proposed network slicing architecture.

Based on the layered design of the proposed scheme, the allocation of RBs to user services is as follows. For users of the ARNs, the LMM module of each ARN initially implements the upper layer of the scheme to monitor the satisfaction grade $S_{m, u}^{\text {estimated }}\left(t_{n}\right)$ of each user service. If the estimated $S_{m, u}^{e s t i m a t e d}\left(t_{n}\right)$ is higher than the predefined $S_{m, u}^{\text {threshold }}$ threshold value, the service requirements can be satisfied from the remaining RBs that exist in the current $A R N_{k}$. Thus, the LAM module of the ARN implements the middle and the lower layers of the slicing scheme to allocate RBs of the $A R N_{k}$ to both GBR and non-GBR services for the next TTI. However, if the estimated satisfaction grade $S_{m, u}^{\text {estimated }}\left(t_{n}\right)$ is lower than the predefined $S_{m, u}^{\text {threshold }}$ threshold value, the service requirements cannot be satisfied from the $R B_{A R N_{k}}^{r e m}\left(t_{n}\right)$. In this case, the ARN borrows extra RBs from the LVRP of the corresponding RSU to achieve the required satisfaction grade. For the users of RSUs, the LMM module of each RSU initially implements the upper layer of the scheme to monitor 
the satisfaction grade $S_{m, u}^{\text {estimated }}\left(t_{n}\right)$ of each user service. If the remaining RBs of the current $R S U_{k}$ can satisfy the predefined $S_{m, u}^{\text {threshold }}$ threshold value, the LAM module of the RSU implements the middle and the lower layers of the slicing scheme to allocate LRBs of the $R S U_{k}$ to both GBR and non-GBR services for the next TTI. The remaining RBs $R B_{k}^{r e m}\left(t_{n}\right)$ are sent back to the VRP of the SDN controller. However, if the remaining RBs of the current $R S U_{k}$ cannot satisfy the $S_{m, u}^{\text {threshold }}$ threshold, the AM of the SDN controller implements the middle and the lower layers of the scheme to allocate extra RBs from the VRP to the user services. As far as the VRP is concerned, the algorithm prefers to allocate RBs with similar sub frequencies for each user to minimize the number of antennas required for each vehicle, which is a factor that affects the energy consumption of the system.

\section{Optimization of the Positions of ARNs}

In this section, an algorithm that optimizes the geographical position of each ARN is described. Using this algorithm, the most appropriate position for each ARN is selected, in order to enhance the overall performance of the system since ARNs with optimized positions provide communication resources with improved channel conditions. More specifically, the main goal of the proposed procedure is the improvement of network technical characteristics such as the throughput, the delay, the jitter and the packet loss ratio, by placing each ARN to the most appropriate position. Indicative criteria that can be considered for this procedure include the vehicle density in each geographical area, the SINR of the corresponding RSU in this area, as well as the average priorities of the services used from the vehicles moving inside this area. Thus, the ARN's position that maximizes these parameters will be selected.

\section{The Icosagonal Fuzzy TOPSIS (IFT)}

As has been proved in several works, the technique for order preference by similarity to ideal solution (TOPSIS) and its variants perform optimal multi-attribute decision making considering multiple criteria. Indicatively, as shown in [43], the trapezoidal fuzzy TOPSIS (TFT) method outperforms the fuzzy analytic hierarchy process (AHP) - elimination et choix traduisant la realité (ELECTRE) [44] method-which is also called FAE method, in scenarios where the most appropriate network should be selected for a user. In addition, as proven in [40], a mobility management scheme that applies the pentagonal fuzzy TOPSIS (PFT) method outperforms the fuzzy AHP simple additive weighting (FAS) [45], the fuzzy AHP multiplicative exponent weighting (FAM) [45] and the FAE methods. Furthermore, similar conclusions about the performance of the TOPSIS method were mentioned to $[46,47]$ against several alternative methodologies. In addition, as it is shown to [48], the trapezoidal fuzzy TOPSIS for heritage route selection (TFT-HRS) method performs the optimal selection of flying routes for drones. By taking into consideration the aforementioned research works, in this paper, the icosagonal fuzzy TOPSIS (IFT) algorithm is proposed for the selection of the optimal positions of the ARNs. It is an improved version of the pentagonal fuzzy TOPSIS (PFT) [40] using interval-valued icosagonal fuzzy numbers for the representations of the values of criteria attributes. As each algorithm that is based on TOPSIS [49], IFT applies the concept that the best alternative solution should have the shortest distance from the positive ideal solution and the longest distance from the negative ideal solution.

Regarding the functionality of the algorithm, a set $C=\left\{C_{1}, C_{2}, \ldots, C_{m}\right\}$ of criteria along with the corresponding criteria weights $w_{1}, w_{2}, \ldots, w_{m}$ are considered. Furthermore, a list $A=\left\{A_{1}, A_{2}, \ldots, A_{z}\right\}$ is constructed determining the alternative positions from which the best one should be selected for the ARN. The steps of the algorithm are as follows: 
- $\quad$ Step 1. Construction of the decision matrix: each $x_{i j}$ element of the $n \times m$ decision matrix $D$ is an interval-valued icosagonal fuzzy number which expresses the evaluation of the alternative position $i$ considering the criterion $j$. Thus:

$$
D=\begin{array}{r|rrr}
\text { ine }_{1} & C_{1} & \ldots & C_{m} \\
x_{11} & \ldots & x_{1 m} \\
\vdots & \vdots & \ddots & \vdots \\
A_{n} & x_{n 1} & \ldots & x_{n m}
\end{array}
$$

where:

$x_{i j}=\left[\left(x_{i j 1}^{U}, x_{i j 2}^{U}, x_{i j 3}^{U}, x_{i j 4}^{U}, x_{i j 5}^{U}, x_{i j 6}^{U}, x_{i j 7}^{U}, x_{i j 8}^{U}, x_{i j 9}^{U}, x_{i j 10}^{U}, x_{i j 11}^{U}, x_{i j 12}^{U}, x_{i j 13}^{U}, x_{i j 14}^{U}, x_{i j 15}^{U}, x_{i j 16}^{U}, x_{i j 17}^{U}, x_{i j 18}^{U}\right.\right.$, $\left.x_{i j 19}^{U}, x_{i j 20}^{U}, v_{i j}^{U}, v_{i j 1}^{U}, v_{i j 2}^{U}, v_{i j 3}^{U}, v_{i j 4}^{U}, v_{i j 5}^{U}, v_{i j 6}^{U}, v_{i j 7}^{U}, v_{i j 8}^{U}\right),\left(x_{i j 1}^{L}, x_{i j 2}^{L}, x_{i j 3}^{L}, x_{i j 4}^{L}, x_{i j 5}^{L}, x_{i j 6}^{L}, x_{i j 7}^{L}, x_{i j 8}^{L}, x_{i j 9}^{L}\right.$, $x_{i j 10}^{L}, x_{i j 11}^{L}, x_{i j 12}^{L}, x_{i j 13}^{L}, x_{i j 14}^{L}, x_{i j 15}^{L}, x_{i j 16}^{L}, x_{i j 17}^{L}, x_{i j 18}^{L}, x_{i j 19}^{L}, x_{i j 20}^{L}, v_{i j}^{L}, v_{i j 1}^{L}, v_{i j 2}^{L}, v_{i j 3}^{L}, v_{i j 4}^{L}, v_{i j 5}^{L}, v_{i j 6}^{L}, v_{i j 7}^{L}$, $\left.\left.v_{i j 8}^{L}\right)\right]$.

- $\quad$ Step 2. Normalization of the decision matrix: consider that $\Omega_{b}$ is the set of benefit evaluation criteria and $\Omega_{c}$ is the set of cost evaluation criteria. Then, the elements of the normalized decision matrix are computed as

$$
\begin{aligned}
& r_{i j}= {\left[\left(\frac{x_{i j 1}^{U}}{b_{j}}, \frac{x_{i j 2}^{U}}{b_{j}}, \frac{x_{i j 3}^{U}}{b_{j}}, \frac{x_{i j 4}^{U}}{b_{j}}, \frac{x_{i j 5}^{U}}{b_{j}}, \frac{x_{i j 6}^{U}}{b_{j}}, \frac{x_{i j 7}^{U}}{b_{j}}, \frac{x_{i j 8}^{U}}{b_{j}}, \frac{x_{i j 9}^{U}}{b_{j}}, \frac{x_{i j 10}^{U}}{b_{j}}, \frac{x_{i j 11}^{U}}{b_{j}}, \frac{x_{i j 12}^{U}}{b_{j}}, \frac{x_{i j 13}^{U}}{b_{j}}, \frac{x_{i j 14}^{U}}{b_{j}}, \frac{x_{i j 15}^{U}}{b_{j}},\right.\right.} \\
&\left.\frac{x_{i j 16}^{U}}{b_{j}}, \frac{x_{i j 17}^{U}}{b_{j}}, \frac{x_{i j 18}^{U}}{b_{j}}, \frac{x_{i j 19}^{U}}{b_{j}}, \frac{x_{i j 20}^{U}}{b_{j}}, v_{i j}^{U}, v_{i j 1}^{U}, v_{i j 2}^{U}, v_{i j 3}^{U}, v_{i j 4}^{U}, v_{i j 5}^{U}, v_{i j 6}^{U}, v_{i j 7}^{U}, v_{i j 8}^{U}\right), \\
&\left(\frac{x_{i j 1}^{L}}{b_{j}}, \frac{x_{i j 2}^{L}}{b_{j}}, \frac{x_{i j 3}^{L}}{b_{j}}, \frac{x_{i j 4}^{L}}{b_{j}}, \frac{x_{i j 5}^{L}}{b_{j}}, \frac{x_{i j 6}^{L}}{b_{j}}, \frac{x_{i j 7}^{L}}{b_{j}}, \frac{x_{i j 8}^{L}}{b_{j}}, \frac{x_{i j 9}^{L}}{b_{j}}, \frac{x_{i j 10}^{L}}{b_{j}}, \frac{x_{i j 11}^{L}}{b_{j}}, \frac{x_{i j 12}^{L}}{b_{j}}, \frac{x_{i j 13}^{L}}{b_{j}}, \frac{x_{i j 14}^{L}}{b_{j}}, \frac{x_{i j 15}^{L}}{b_{j}},\right. \\
&\left.\left.\frac{x_{i j 16}^{L}}{b_{j}}, \frac{x_{i j 17}^{L}}{b_{j}}, \frac{x_{i j 18}^{L}}{b_{j}}, \frac{x_{i j 19}^{L}}{b_{j}}, \frac{x_{i j 20}^{L}}{b_{j}}, v_{i j}^{L}, v_{i j 1}^{L}, v_{i j 2}^{L}, v_{i j 3}^{L}, v_{i j 4}^{L}, v_{i j 5}^{L}, v_{i j 6}^{L}, v_{i j 7}^{L}, v_{i j 8}^{L}\right)\right]
\end{aligned}
$$

where $b_{j}=\max _{i} x_{i j}^{U}$ for each $j \in \Omega_{b}$, or:

$$
\begin{aligned}
& r_{i j}= {\left[\left(\frac{c_{j}}{x_{i j 1}^{U}}, \frac{c_{j}}{x_{i j 2}^{U}}, \frac{c_{j}}{x_{i j 3}^{U}}, \frac{c_{j}}{x_{i j 4}^{U}}, \frac{c_{j}}{x_{i j 5}^{U}}, \frac{c_{j}}{x_{i j 6}^{U}}, \frac{c_{j}}{x_{i j 7}^{U}}, \frac{c_{j}}{x_{i j 8}^{U}}, \frac{c_{j}}{x_{i j 9}^{U}}, \frac{c_{j}}{x_{i j 10}^{U}}, \frac{c_{j}}{x_{i j 11}^{U}}, \frac{c_{j}}{x_{i j 12}^{U}}, \frac{c_{j}}{x_{i j 13}^{U}}, \frac{c_{j}}{x_{i j 14}^{U}}, \frac{c_{j}}{x_{i j 15}^{U}},\right.\right.} \\
&\left.\frac{c_{j}}{x_{i j 16}^{U}}, \frac{c_{j}}{x_{i j 17}^{U}}, \frac{c_{j}}{x_{i j 18}^{U}}, \frac{c_{j}}{x_{i j 19}^{U}}, \frac{c_{j}}{x_{i j 20}^{U}}, v_{i j}^{U}, v_{i j 1}^{U}, v_{i j 2}^{U} v_{i j 3}^{U}, v_{i j 4}^{U}, v_{i j 5}^{U}, v_{i j 6}^{U}, v_{i j 7}^{U}, v_{i j 8}^{U}\right), \\
&\left(\frac{c_{j}}{x_{i j 1}^{L}}, \frac{c_{j}}{x_{i j 2}^{L}}, \frac{c_{j}}{x_{i j 3}^{L}}, \frac{c_{j}}{x_{i j 4}^{L}}, \frac{c_{j}}{x_{i j 5}^{L}}, \frac{c_{j}}{x_{i j 6}^{L}}, \frac{c_{j}}{x_{i j 7}^{L}}, \frac{c_{j}}{x_{i j 8}^{L}}, \frac{c_{j}}{x_{i j 9}^{L}}, \frac{c_{j}}{x_{i j 10}^{L}}, \frac{c_{j}}{x_{i j 11}^{L}}, \frac{c_{j}}{x_{i j 12}^{L}}, \frac{c_{j}}{x_{i j 13}^{L}}, \frac{c_{j}}{x_{i j 14}^{L}}, \frac{c_{j}}{x_{i j 15}^{L}},\right. \\
&\left.\left.\frac{c_{j}}{x_{i j 16}^{L}}, \frac{c_{j}}{x_{i j 17}^{L}}, \frac{c_{j}}{x_{i j 18}^{L}}, \frac{c_{j}}{x_{i j 19}^{L}}, \frac{c_{j}}{x_{i j 20}^{L}}, v_{i j}^{L}, v_{i j 1}^{L}, v_{i j 2}^{L}, v_{i j 3}^{L}, v_{i j 4}^{L}, v_{i j 5}^{L}, v_{i j 6}^{L}, v_{i j 7}^{L}, v_{i j 8}^{L}\right)\right]
\end{aligned}
$$

where $c_{j}=\min _{i} x_{i j}^{L}$ for each $j \in \Omega_{c}$.

- $\quad$ Step 3. Construction of the weighted normalized decision matrix: for the construction of the weighted normalized decision matrix, each element $r_{i j}$ of the normalized decision matrix is multiplied with the respective criterion weight $w_{j}$ according to the formula:

$$
\begin{gathered}
u_{i j}=\left[\left(r_{i j 1}^{U} \cdot w_{j}, r_{i j 2}^{U} \cdot w_{j}, r_{i j 3}^{U} \cdot w_{j}, r_{i j 4}^{U} \cdot w_{j}, r_{i j 5}^{U} \cdot w_{j}, r_{i j 6}^{U} \cdot w_{j}, r_{i j 7}^{U} \cdot w_{j}, r_{i j 8}^{U} \cdot w_{j}, r_{i j 9}^{U} \cdot w_{j},\right.\right. \\
r_{i j 10}^{U} \cdot w_{j}, r_{i j 11}^{U} \cdot w_{j}, r_{i j 12}^{U} \cdot w_{j}, r_{i j 13}^{U} \cdot w_{j}, r_{i j 14}^{U} \cdot w_{j}, r_{i j 15}^{U} \cdot w_{j}, r_{i j 16}^{U} \cdot w_{j}, r_{i j 17}^{U} \cdot w_{j}, r_{i j 18}^{U} \cdot w_{j}, \\
\left.r_{i j 19}^{U} \cdot w_{j}, r_{i j 20}^{U} \cdot w_{j}, v_{i j}^{U}, v_{i j 1}^{U}, v_{i j 2}^{U}, v_{i j 3}^{U}, v_{i j 4}^{U}, v_{i j 5}^{U}, v_{i j 6}^{U}, v_{i j 7}^{U}, v_{i j 8}^{U}\right), \\
\left(r_{i j 1}^{L} \cdot w_{j}, r_{i j 2}^{L} \cdot w_{j}, r_{i j 3}^{L} \cdot w_{j}, r_{i j 4}^{L} \cdot w_{j}, r_{i j 5}^{L} \cdot w_{j}, r_{i j 6}^{L} \cdot w_{j}, r_{i j 7}^{L} \cdot w_{j}, r_{i j 8}^{L} \cdot w_{j}, r_{i j 9}^{L} \cdot\right. \\
w_{j}, r_{i j 10}^{L} \cdot w_{j}, r_{i j 11}^{L} \cdot w_{j}, r_{i j 12}^{L} \cdot w_{j}, r_{i j 13}^{L} \cdot w_{j}, r_{i j 14}^{L} \cdot w_{j}, r_{i j 15}^{L} \cdot w_{j}, r_{i j 16}^{L} \cdot w_{j}, r_{i j 17}^{L} \cdot w_{j}, r_{i j 18}^{L} \cdot \\
\left.\left.w_{j}, r_{i j 19}^{L} \cdot w_{j}, r_{i j 20}^{L} \cdot w_{j}, v_{i j}^{L}, v_{i j 1}^{L}, v_{i j 2}^{L}, v_{i j 3}^{L}, v_{i j 4}^{L}, v_{i j 5}^{L}, v_{i j 6}^{L}, v_{i j 7}^{L}, v_{i j 8}^{L}\right)\right]
\end{gathered}
$$


At this point, it must be noted that the criteria weights can be estimated using the analytic hierarchy process (AHP) [50], the analytic network process (ANP) [51] or another similar method.

- Step 4. Determination of the positive and negative ideal solution: in this step, the positive ideal solution is defined as

$$
\begin{aligned}
& X^{+}=\left[\left(x_{i j 1}^{+U}, x_{i j 2}^{+U}, x_{i j 3}^{+U}, x_{i j 4}^{+U}, x_{i j 5}^{+U}, x_{i j 6}^{+U}, x_{i j 7}^{+U}, x_{i j 8}^{+U}, x_{i j 9}^{+U}, x_{i j 10}^{+U}, x_{i j 11}^{+U}, x_{i j 12}^{+U}, x_{i j 13}^{+U}, x_{i j 14}^{+U}, x_{i j 15}^{+U},\right.\right. \\
& \left.x_{i j 16}^{+U}, x_{i j 17}^{+U}, x_{i j 18}^{+U}, x_{i j 19}^{+U}, x_{i j 20}^{+U}, v_{i j}^{+U}, v_{i j 1}^{+U}, v_{i j 2}^{+U}, v_{i j 3}^{+U}, v_{i j 4}^{+U}, v_{i j 5}^{+U}, v_{i j 6}^{+U}, v_{i j 7}^{+U}, v_{i j 8}^{+U}\right), \\
& \left(x_{i j 1}^{+L}, x_{i j 2}^{+L}, x_{i j 3}^{+L}, x_{i j 4}^{+L}, x_{i j 5}^{+L}, x_{i j 6}^{+L}, x_{i j 7}^{+L}, x_{i j 8}^{+L}, x_{i j 9}^{+L}, x_{i j 10}^{+L}, x_{i j 11}^{+L}, x_{i j 12}^{+L}, x_{i j 13}^{+L}, x_{i j 14}^{+L}, x_{i j 15}^{+L}\right. \text {, } \\
& \left.\left.x_{i j 16}^{+L}, x_{i j 17}^{+L}, x_{i j 18}^{+L}, x_{i j 19}^{+L}, x_{i j 20}^{+L}, v_{i j}^{+L}, v_{i j 1}^{+L}, v_{i j 2}^{+L}, v_{i j 3}^{+L}, v_{i j 4}^{+L}, v_{i j 5}^{+L}, v_{i j 6}^{+L}, v_{i j 7}^{+L}, v_{i j 8}^{+L}\right)\right]= \\
& {\left[\left(\bigwedge_{i} u_{i j 1}^{U}, \bigwedge_{i} u_{i j 2}^{U}, \bigwedge_{i} u_{i j 3}^{U}, \bigwedge_{i} u_{i j 4}^{U}, \bigwedge_{i} u_{i j 5}^{U}, \bigwedge_{i} u_{i j 6}^{U}, \bigwedge_{i} u_{i j 7}^{U}, \bigwedge_{i} u_{i j 8}^{U}, \bigwedge_{i} u_{i j 9}^{U}, \bigwedge_{i} u_{i j 10}^{U},\right.\right.} \\
& \bigwedge_{i} u_{i j 11}^{U}, \bigwedge_{i} u_{i j 12}^{U}, \bigwedge_{i} u_{i j 13}^{U}, \bigwedge_{i} u_{i j 14}^{U}, \bigwedge_{i} u_{i j 15}^{U}, \bigwedge_{i} u_{i j 16}^{U}, \bigwedge_{i} u_{i j 17}^{U}, \bigwedge_{i} u_{i j 18}^{U}, \bigwedge_{i} u_{i j 19}^{U}, \bigwedge_{i} u_{i j 20}^{U}, \\
& \left.v_{i j}^{U}, v_{i j 1}^{U}, v_{i j 2}^{U}, v_{i j 3}^{U}, v_{i j 4}^{U}, v_{i j 5}^{U}, v_{i j 6}^{U}, v_{i j 7}^{U}, v_{i j 8}^{U}\right), \\
& \left(\bigwedge_{i} u_{i j 1}^{L}, \bigwedge_{i} u_{i j 2}^{L}, \bigwedge_{i} u_{i j 3}^{L}, \bigwedge_{i} u_{i j 4}^{L}, \bigwedge_{i} u_{i j 5}^{L}, \bigwedge_{i} u_{i j 6}^{L}, \bigwedge_{i} u_{i j 7}^{L}, \bigwedge_{i} u_{i j 8}^{L}, \bigwedge_{i} u_{i j 9}^{L}, \bigwedge_{i} u_{i j 10}^{L},\right. \\
& \bigwedge_{i} u_{i j 11}^{L}, \bigwedge_{i} u_{i j 12}^{L}, \bigwedge_{i} u_{i j 13}^{L}, \bigwedge_{i} u_{i j 14}^{L}, \bigwedge_{i} u_{i j 15}^{L}, \bigwedge_{i} u_{i j 16}^{L}, \bigwedge_{i} u_{i j 17}^{L}, \bigwedge_{i} u_{i j 18}^{L}, \bigwedge_{i} u_{i j 19}^{L}, \bigwedge_{i} u_{i j 20}^{L}, \\
& \left.\left.v_{i j}^{L}, v_{i j 1}^{L}, v_{i j 2}^{L}, v_{i j 3}^{L}, v_{i j 4}^{L}, v_{i j 5}^{L}, v_{i j 6}^{L}, v_{i j 7}^{L}, v_{i j 8}^{L}\right)\right]
\end{aligned}
$$

where $\bigwedge_{i} \equiv \max _{i}$ in case $j \in \Omega_{b}$ and $\bigwedge_{i} \equiv \min _{i}$ in case $j \in \Omega_{c}$. Accordingly, the negative ideal solutions is defined as

$$
\begin{aligned}
& X^{-}=\left[\left(x_{i j 1}^{-U}, x_{i j 2}^{-U}, x_{i j 3}^{-U}, x_{i j 4}^{-U}, x_{i j 5}^{-u}, x_{i j 6}^{-U}, x_{i j 7}^{-U}, x_{i j 8}^{-U}, x_{i j 9}^{-u}, x_{i j 10}^{-U}, x_{i j 11}^{-U}, x_{i j 12}^{-U}, x_{i j 13}^{-U}, x_{i j 14}^{-U}, x_{i j 15}^{-U},\right.\right. \\
& \left.x_{i j 16}^{-U}, x_{i j 17}^{-U}, x_{i j 18}^{-U}, x_{i j 19}^{-U}, x_{i j 20}^{-U}, v_{i j}^{-U}, v_{i j 1}^{-U}, v_{i j 2}^{-U}, v_{i j 3}^{-U}, v_{i j 4}^{-U}, v_{i j 5}^{-U}, v_{i j 6}^{-U}, v_{i j 7}^{-U}, v_{i j 8}^{-U}\right), \\
& \left(x_{i j 1}^{-L}, x_{i j 2}^{-L}, x_{i j 3}^{-L}, x_{i j 4}^{-L}, x_{i j 5}^{-L}, x_{i j 6}^{-L}, x_{i j 7}^{-L}, x_{i j 8}^{-L}, x_{i j 9}^{-L}, x_{i j 10}^{-L}, x_{i j 11}^{-L}, x_{i j 12}^{-L}, x_{i j 13}^{-L}, x_{i j 14}^{-L}, x_{i j 15}^{-L}\right. \text {, } \\
& \left.\left.x_{i j 16}^{-L}, x_{i j 17}^{-L}, x_{i j 18}^{-L}, x_{i j 19}^{-L}, x_{i j 20}^{-L}, v_{i j}^{-L}, v_{i j 1}^{-L}, v_{i j 2}^{-L}, v_{i j 3}^{-L}, v_{i j 4}^{-L}, v_{i j 5}^{-L}, v_{i j 6}^{-L}, v_{i j 7}^{-L}, v_{i j 8}^{-L}\right)\right]= \\
& {\left[\left(\bigvee_{i} u_{i j 1}^{U}, \bigvee_{i} u_{i j 2}^{u}, \bigvee_{i} u_{i j 3}^{u}, \bigvee_{i} u_{i j 4}^{u}, \bigvee_{i} u_{i j 5}^{U}, \bigvee_{i} u_{i j 6}^{U}, \bigvee_{i} u_{i j 7}^{U}, \bigvee_{i} u_{i j \delta}^{u}, \bigvee_{i} u_{i j 9}^{u}, \bigvee_{i} u_{i j 10}^{U}\right.\right.} \\
& \bigvee_{i} u_{i j 11}^{u}, \bigvee_{i} u_{i j 12}^{u}, \bigvee_{i} u_{i j 13}^{U}, \bigvee_{i} u_{i j 14}^{u}, \bigvee_{i} u_{i j 15}^{U}, \bigvee_{i} u_{i j 16}^{u}, \bigvee_{i} u_{i j 17}^{U}, \bigvee_{i} u_{i j 18}^{U}, \bigvee_{i} u_{i j 19}^{U}, \bigvee_{i} u_{i j 20}^{u} \\
& \left.v_{i j}^{U}, v_{i j 1}^{U}, v_{i j 2}^{U}, v_{i j 3}^{U}, v_{i j 4}^{U}, v_{i j,}^{U}, v_{i j 6}^{U}, v_{i j 7}^{U}, v_{i j 8}^{U}\right) \text {, } \\
& \left(\bigvee_{i} u_{i j 1}^{L}, \bigvee_{i} u_{i j 2}^{L}, \bigvee_{i} u_{i j 3}^{L}, \bigvee_{i} u_{i j 4}^{L}, \bigvee_{i} u_{i j 5}^{L}, \bigvee_{i} u_{i j 6}^{L}, \bigvee_{i} u_{i j 7}^{L}, \bigvee_{i} u_{i j 8}^{L}, \bigvee_{i} u_{i j 9}^{L}, \bigvee_{i} u_{i j 10}^{L},\right. \\
& \bigvee u_{i j 11}^{L}, \bigvee u_{i j 12}^{L}, \bigvee u_{i j 11}^{L}, \bigvee u_{i j 11}^{L}, \bigvee u_{i j 15}^{L}, \bigvee u_{i j 16}^{L}, \bigvee u_{i j 17}^{L}, \bigvee u_{i j 18}^{L}, \bigvee u_{i j 19}^{L}, \bigvee u_{i j 20}^{L}, \\
& \left.\left.v_{i j}^{L}, v_{i j 1}^{L}, v_{i j 2}^{L}, v_{i j 3}^{L}, v_{i j 4}^{L}, v_{i j 5}^{L}, v_{i j 6}^{L}, v_{i j 7}^{L}, v_{i j 8}^{L}\right)\right]
\end{aligned}
$$

where $\bigvee_{i} \equiv \min _{i}$ in case $j \in \Omega_{b}$ and $\bigvee_{i} \equiv \max _{i}$ in case $j \in \Omega_{c}$.

- Step 5. Estimation of the distance of each alternative solution from the positive and the negative ideal solutions: the distances $d_{i 1}^{+}$and $d_{i 2}^{+}$of each alternative solution from the positive ideal solution are estimated as follows:

$$
\begin{aligned}
d_{i 1}^{+}=\sum_{j=1}^{m}\{ & \frac{1}{20}\left[\left(u_{i j 1}^{U}-x_{i j 1}^{+U}\right)^{2}+\left(u_{i j 2}^{U}-x_{i j 2}^{+U}\right)^{2}+\left(u_{i j 3}^{U}-x_{i j 3}^{+U}\right)^{2}+\left(u_{i j 4}^{U}-x_{i j 4}^{+U}\right)^{2}\right. \\
& +\left(u_{i j 5}^{U}-x_{i j 5}^{+U}\right)^{2}+\left(u_{i j 6}^{U}-x_{i j 6}^{+U}\right)^{2}+\left(u_{i j 7}^{U}-x_{i j 7}^{+U}\right)^{2}+\left(u_{i j 8}^{U}-x_{i j 8}^{+U}\right)^{2} \\
+ & \left(u_{i j 9}^{U}-x_{i j 9}^{+U}\right)^{2}+\left(u_{i j 10}^{U}-x_{i j 10}^{+U}\right)^{2}+\left(u_{i j 11}^{U}-x_{i j 11}^{+U}\right)^{2}+\left(u_{i j 12}^{U}-x_{i j 12}^{+U}\right)^{2} \\
+ & \left(u_{i j 13}^{U}-x_{i j 13}^{+U}\right)^{2}+\left(u_{i j 14}^{U}-x_{i j 14}^{+U}\right)^{2}+\left(u_{i j 15}^{U}-x_{i j 15}^{+U}\right)^{2}+\left(u_{i j 16}^{U}-x_{i j 16}^{+U}\right)^{2} \\
& \left.\left.+\left(u_{i j 17}^{U}-x_{i j 17}^{+U}\right)^{2}+\left(u_{i j 18}^{U}-x_{i j 18}^{+U}\right)^{2}+\left(u_{i j 19}^{U}-x_{i j 19}^{+U}\right)^{2}+\left(u_{i j 20}^{U}-x_{i j 20}^{+U}\right)^{2}\right]\right\}^{\frac{1}{2}}
\end{aligned}
$$




$$
\begin{aligned}
d_{i 2}^{+}=\sum_{j=1}^{m}\{ & \frac{1}{20}\left[\left(u_{i j 1}^{L}-x_{i j 1}^{+L}\right)^{2}+\left(u_{i j 2}^{L}-x_{i j 2}^{+L}\right)^{2}+\left(u_{i j 3}^{L}-x_{i j 3}^{+L}\right)^{2}+\left(u_{i j 4}^{L}-x_{i j 1}^{+L}\right)^{2}\right. \\
& +\left(u_{i j 5}^{L}-x_{i j 5}^{+L}\right)^{2}+\left(u_{i j 6}^{L}-x_{i j 6}^{+L}\right)^{2}+\left(u_{i j 7}^{L}-x_{i j 7}^{+L}\right)^{2}+\left(u_{i j 8}^{L}-x_{i j 8}^{+L}\right)^{2} \\
+ & \left(u_{i j 9}^{L}-x_{i j 9}^{+L}\right)^{2}+\left(u_{i j 10}^{L}-x_{i j 10}^{+L}\right)^{2}+\left(u_{i j 11}^{L}-x_{i j 11}^{+L}\right)^{2}+\left(u_{i j 12}^{L}-x_{i j 12}^{+L}\right)^{2} \\
+ & \left(u_{i j 13}^{L}-x_{i j 13}^{+L}\right)^{2}+\left(u_{i j 14}^{L}-x_{i j 14}^{+L}\right)^{2}+\left(u_{i j 15}^{L}-x_{i j 15}^{+L}\right)^{2}+\left(u_{i j 16}^{L}-x_{i j 16}^{+L}\right)^{2} \\
& \left.\left.+\left(u_{i j 17}^{L}-x_{i j 17}^{+L}\right)^{2}+\left(u_{i j 18}^{L}-x_{i j 18}^{+L}\right)^{2}+\left(u_{i j 19}^{L}-x_{i j 19}^{+L}\right)^{2}+\left(u_{i j 20}^{L}-x_{i j 20}^{+L}\right)^{2}\right]\right\}^{\frac{1}{2}}
\end{aligned}
$$

Similarly, the distances $d_{i 1}^{-}$and $d_{i 2}^{-}$of each alternative solution from the negative ideal solution are estimated as follows:

$$
\begin{aligned}
& d_{i 1}^{-}=\sum_{j=1}^{m}\left\{\frac { 1 } { 2 0 } \left[\left(u_{i j 1}^{U}-x_{i j 1}^{-U}\right)^{2}+\left(u_{i j 2}^{U}-x_{i j 2}^{-U}\right)^{2}+\left(u_{i j 3}^{U}-x_{i j 3}^{-U}\right)^{2}+\left(u_{i j 4}^{U}-x_{i j 4}^{-U}\right)^{2}\right.\right. \\
& +\left(u_{i j 5}^{U}-x_{i j 5}^{-U}\right)^{2}+\left(u_{i j 6}^{U}-x_{i j 6}^{-U}\right)^{2}+\left(u_{i j 7}^{U}-x_{i j 7}^{-U}\right)^{2}+\left(u_{i j 8}^{U}-x_{i j 8}^{-U}\right)^{2} \\
& +\left(u_{i j 9}^{U}-x_{i j 9}^{-U}\right)^{2}+\left(u_{i j 10}^{U}-x_{i j 10}^{-U}\right)^{2}+\left(u_{i j 11}^{U}-x_{i j 11}^{-U}\right)^{2}+\left(u_{i j 12}^{U}-x_{i j 12}^{-U}\right)^{2} \\
& +\left(u_{i j 13}^{U}-x_{i j 13}^{-U}\right)^{2}+\left(u_{i j 14}^{U}-x_{i j 14}^{-U}\right)^{2}+\left(u_{i j 15}^{U}-x_{i j 15}^{-U}\right)^{2}+\left(u_{i j 16}^{U}-x_{i j 16}^{-U}\right)^{2} \\
& \left.\left.+\left(u_{i j 17}^{U}-x_{i j 17}^{-U}\right)^{2}+\left(u_{i j 18}^{U}-x_{i j 18}^{-U}\right)^{2}+\left(u_{i j 19}^{U}-x_{i j 19}^{-U}\right)^{2}+\left(u_{i j 20}^{U}-x_{i j 20}^{-U}\right)^{2}\right]\right\}^{\frac{1}{2}} \\
& d_{i 2}^{-}=\sum_{j=1}^{m}\left\{\frac { 1 } { 2 0 } \left[\left(u_{i j 1}^{L}-x_{i j 1}^{-L}\right)^{2}+\left(u_{i j 2}^{L}-x_{i j 2}^{-L}\right)^{2}+\left(u_{i j 3}^{L}-x_{i j 3}^{-L}\right)^{2}+\left(u_{i j 4}^{L}-x_{i j 4}^{-L}\right)^{2}\right.\right. \\
& +\left(u_{i j 5}^{L}-x_{i j 5}^{-L}\right)^{2}+\left(u_{i j 6}^{L}-x_{i j 6}^{-L}\right)^{2}+\left(u_{i j 7}^{L}-x_{i j 7}^{-L}\right)^{2}+\left(u_{i j 8}^{L}-x_{i j 8}^{-L}\right)^{2} \\
& +\left(u_{i j 9}^{L}-x_{i j 9}^{-L}\right)^{2}+\left(u_{i j 10}^{L}-x_{i j 10}^{-L}\right)^{2}+\left(u_{i j 11}^{L}-x_{i j 11}^{-L}\right)^{2}+\left(u_{i j 12}^{L}-x_{i j 12}^{-L}\right)^{2} \\
& +\left(u_{i j 13}^{L}-x_{i j 13}^{-L}\right)^{2}+\left(u_{i j 14}^{L}-x_{i j 14}^{-L}\right)^{2}+\left(u_{i j 15}^{L}-x_{i j 15}^{-L}\right)^{2}+\left(u_{i j 16}^{L}-x_{i j 16}^{-L}\right)^{2} \\
& \left.\left.+\left(u_{i j 17}^{L}-x_{i j 17}^{-L}\right)^{2}+\left(u_{i j 18}^{L}-x_{i j 18}^{-L}\right)^{2}+\left(u_{i j 19}^{L}-x_{i j 19}^{-L}\right)^{2}+\left(u_{i j 20}^{L}-x_{i j 20}^{-L}\right)^{2}\right]\right\}^{\frac{1}{2}}
\end{aligned}
$$

Thus, the distance of the alternative solutions from the positive and negative ideal solutions are expressed by intervals such as $\left[d_{i 1}^{+}, d_{i 2}^{+}\right]$and $\left[d_{i 1}^{-}, d_{i 2}^{-}\right][52]$, instead of single values. In this way, less information is lost.

- Step 6. Calculation of the relative closeness: the relative closeness of the distances from the ideal solutions are computed as follows:

$$
R C_{i 1}=\frac{d_{i 1}^{-}}{d_{i 1}^{+}+d_{i 1}^{-}}
$$

and:

$$
R C_{i 2}=\frac{d_{i 2}^{-}}{d_{i 2}^{+}+d_{i 2}^{-}}
$$

The compound relative closeness is obtained from the average of the above values:

$$
R C_{i}=\frac{R C_{i 1}+R C_{i 2}}{2}
$$

- Step 7. Alternatives ranking: the alternative solutions are ranked according to their $R C_{i}$ values. The position with the higher $R C_{i}$ value is selected since it is considered the best alternative solution.

\section{Simulation Setup}

For the evaluation of the proposed scheme, the UAV-aided 5G network topology presented in Figure 4 is simulated. A map of the area where the University of Piraeus is 
located has been created using the open street map (OSM) software [53]. Then, the map has been used as input in the simulator of urban mobility (SUMO) simulator [54] allowing the production of a trace file indicating the mobility of vehicles in the corresponding geographical area. At this point, it has to be noted that the average height of the buildings that exist to the simulated map, is supposed to be equal to $30 \mathrm{~m}$. Subsequently, the network topology is being built upon the map, using the Network Simulator 3 (NS3) [55], including a Fog and a Cloud infrastructure.

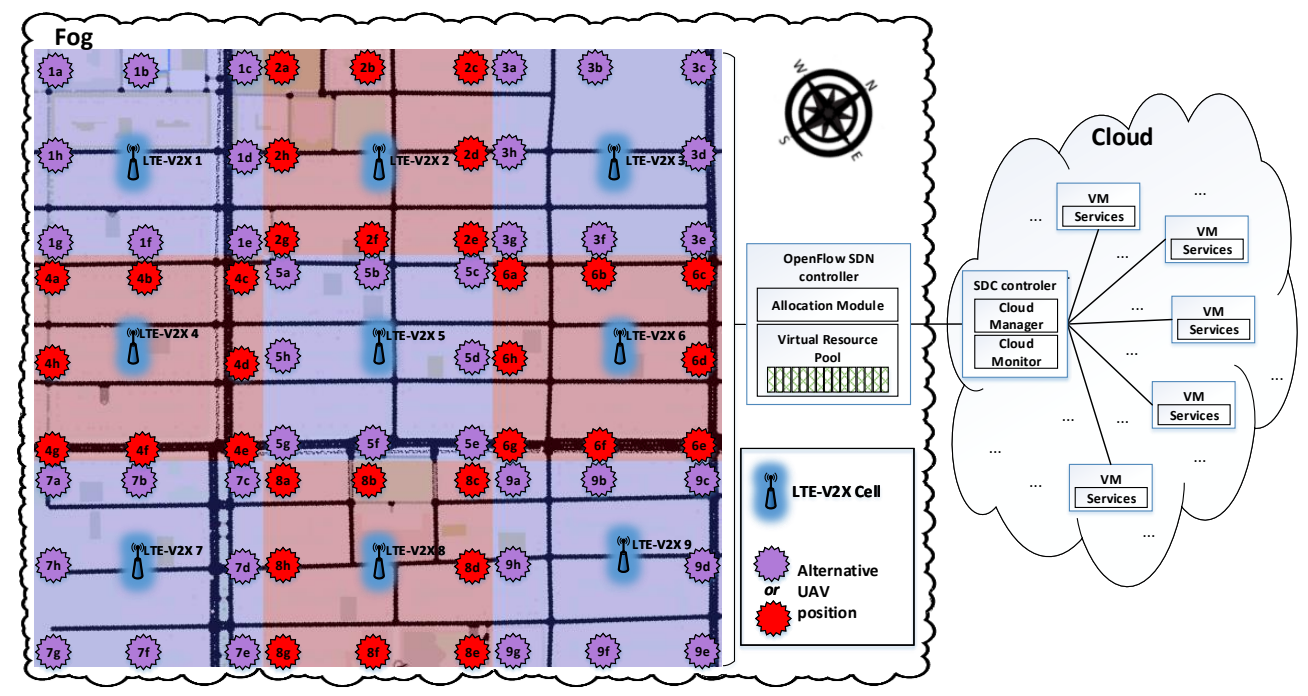

Figure 4. The simulated topology.

The Fog infrastructure consists of nine LTE-V2X RSUs, along with an ARN per LTEV2X RSU providing additional communication resources for users moving inside the corresponding coverage area. Both the medium access control (MAC) and the physical (PHY) layers of the LTE-V2X RSUs and ARNs are simulated using the LTE-V2X extension module of the NS3 [56]. In addition, the default altitude of each ARN is equal to the average height of the buildings that exist on the map. Table 3 presents the spectrum used from each RSU and ARN, while Table 4 presents their geographical positions. Also, an OpenFlow SDN controller provides centralized control of the entire network access environment. The 3GPP urban channel model $[57,58]$ is considered. Due to the assumption that the channel between LTE-V2X RSU or ARN and the vehicular user usually encounters nonline-of-sight (NLOS) transmission, its propagation loss is estimated using Formulas (22) and (23), respectively, where $d$ represents the distance among the nodes and $f_{c}$ represents the carrier frequency. Correspondingly, due to the assumption that the channel between a LTE-V2X RSU and an ARN encounters line-of-sight (LOS) transmission, its propagation loss is estimated using Formula (24). Additionally, it should be noted that when the battery level of an ARN tends to run out, another ARN replaces it in the corresponding position. In this case, the respective users perform a handover from the previous to the new ARN:

$$
\begin{gathered}
P L_{L T E-V 2 X R S U \rightarrow \text { VehicularUser }}^{N L O S}=37.6 \cdot \log _{10}(d)+58.94+21 \cdot \log _{10}\left(f_{c}\right) \\
P L_{A R N \rightarrow \text { Vehicular } U \text { ser }}^{N L O S}=36.7 \cdot \log _{10}(d)+22.7+26 \cdot \log _{10}\left(f_{c}\right) \\
P L_{L T E-V 2 X R S U \rightarrow A R N}^{L O S}=22 \cdot \log _{10}(d)+28+20 \cdot \log _{10}\left(f_{c}\right)
\end{gathered}
$$

Accordingly, the Cloud infrastructure includes a set of virtual machines (VMs) providing services such as autonomous navigation (ANav), conversational voice (CVo), conversational video (CVi) and Web browsing (WB). Table 5 presents the $5 \mathrm{QI}$ value assigned to each service as defined in the 5GPPP specifications for 5G communications [39], along with the corresponding constrains that should be satisfied. 
Table 3. The spectrum used from each RSU and ARN.

\begin{tabular}{cccccc}
\hline \multirow{2}{*}{ Name } & \multicolumn{2}{c}{ Uplink Spectrum } & \multicolumn{2}{c}{ Downlink Spectrum } & \multirow{2}{*}{ LTE Band Number } \\
\cline { 2 - 5 } & From & To & From & To & 28 \\
\hline LTE-V2X 1 & $703 \mathrm{MHz}$ & $723 \mathrm{MHz}$ & $758 \mathrm{MHz}$ & $778 \mathrm{MHz}$ & 20 \\
LTE-V2X 2 & $832 \mathrm{MHz}$ & $852 \mathrm{MHz}$ & $800 \mathrm{MHz}$ & $820 \mathrm{MHz}$ & 24 \\
LTE-V2X 3 & $1630 \mathrm{MHz}$ & $1650 \mathrm{MHz}$ & $1525 \mathrm{MHz}$ & $1545 \mathrm{MHz}$ & 3 \\
LTE-V2X 4 & $1710 \mathrm{MHz}$ & $1730 \mathrm{MHz}$ & $1805 \mathrm{MHz}$ & $1825 \mathrm{MHz}$ & 3 \\
LTE-V2X 5 & $1730 \mathrm{MHz}$ & $1750 \mathrm{MHz}$ & $1825 \mathrm{MHz}$ & $1845 \mathrm{MHz}$ & 3 \\
LTE-V2X 6 & $1750 \mathrm{MHz}$ & $1770 \mathrm{MHz}$ & $1845 \mathrm{MHz}$ & $1865 \mathrm{MHz}$ & 15 \\
LTE-V2X 7 & $1900 \mathrm{MHz}$ & $1920 \mathrm{MHz}$ & $2600 \mathrm{MHz}$ & $2620 \mathrm{MHz}$ & 1 \\
LTE-V2X 8 & $1920 \mathrm{MHz}$ & $1940 \mathrm{MHz}$ & $2110 \mathrm{MHz}$ & $2130 \mathrm{MHz}$ & 1 \\
LTE-V2X 9 & $1940 \mathrm{MHz}$ & $1960 \mathrm{MHz}$ & $2130 \mathrm{MHz}$ & $2150 \mathrm{MHz}$ & 1 \\
ARN 1 & $1960 \mathrm{MHz}$ & $1980 \mathrm{MHz}$ & $2150 \mathrm{MHz}$ & $2170 \mathrm{MHz}$ & 23 \\
ARN 2 & $2000 \mathrm{MHz}$ & $2020 \mathrm{MHz}$ & $2180 \mathrm{MHz}$ & $2200 \mathrm{MHz}$ & 7 \\
ARN 3 & $2500 \mathrm{MHz}$ & $2520 \mathrm{MHz}$ & $2620 \mathrm{MHz}$ & $2640 \mathrm{MHz}$ & 7 \\
ARN 4 & $2520 \mathrm{MHz}$ & $2540 \mathrm{MHz}$ & $2640 \mathrm{MHz}$ & $2660 \mathrm{MHz}$ & 7 \\
ARN 5 & $2540 \mathrm{MHz}$ & $2560 \mathrm{MHz}$ & $2660 \mathrm{MHz}$ & $2680 \mathrm{MHz}$ & 22 \\
ARN 6 & $3410 \mathrm{MHz}$ & $3430 \mathrm{MHz}$ & $3510 \mathrm{MHz}$ & $3530 \mathrm{MHz}$ & 22 \\
ARN 7 & $3430 \mathrm{MHz}$ & $3450 \mathrm{MHz}$ & $3530 \mathrm{MHz}$ & $3550 \mathrm{MHz}$ & 22 \\
ARN 8 & $3450 \mathrm{MHz}$ & $3470 \mathrm{MHz}$ & $3550 \mathrm{MHz}$ & $3570 \mathrm{MHz}$ & 22 \\
ARN 9 & $3470 \mathrm{MHz}$ & $3490 \mathrm{MHz}$ & $3570 \mathrm{MHz}$ & $3590 \mathrm{MHz}$ & \\
\hline
\end{tabular}

Table 4. The geographical position of each RSU and ARN.

\begin{tabular}{|c|c|}
\hline RSU & Geographic Latitude, Geographic Longitude \\
\hline LTE-V2X 1 & $37.941746,23.647962$ \\
\hline LTE-V2X 2 & $37.942985,23.649007$ \\
\hline LTE-V2X 3 & $37.944259,23.650166$ \\
\hline LTE-V2X 4 & $37.941141,23.649080$ \\
\hline LTE-V2X 5 & $37.942303,23.650272$ \\
\hline LTE-V2X 6 & $37.943664,23.651470$ \\
\hline LTE-V2X 7 & $37.940304,23.650710$ \\
\hline LTE-V2X 8 & $37.941564,23.651784$ \\
\hline LTE-V2X 9 & $37.942847,23.652859$ \\
\hline ARN & Possible Position: Geographic Latitude, Geographic Longitude \\
\hline ARN 1 & $\begin{array}{l}\text { 1a: } 37.941630,23.646994 \text { - 1b: } 37.942107,23.647428 \text { - 1c: } 37.942617,23.647842 \text { - 1d: } 37.942334,23.648342 \\
\text { 1e: } 37.941989,23.648996 \text { - 1f: } 37.941489,23.648520 \text { - 1g: } 37.941061,23.648098 \text { - 1h: } 37.941347,23.647473\end{array}$ \\
\hline ARN 2 & $\begin{array}{l}\text { 2a: } 37.942795,23.647994 \text { - 2b: 37.943256, } 23.648375 \text { - 2c: } 37.943846,23.648896 \text { - 2d: } 37.942317,23.648341 \\
\text { 2e: } 37.943244,23.650030 \text { - 2f: 37.942758, } 23.649617 \text { - 2g: } 37.942224,23.649160 \text { - 2h: } 37.942592,23.648570\end{array}$ \\
\hline ARN 3 & $\begin{array}{l}\text { 3a: } 37.944008,23.649037 \text { - 3b: 37.944502, } 23.649430 \text { - 3c: 37.945053, 23.649887 - 3d: } 37.944801,23.650429 \\
\text { 3e: } 37.944441,23.651041 \text { - 3f: 37.943850, } 23.650572 \text { - 3g: 37.943340, 23.650169 - 3h: } 37.943895,23.649658\end{array}$ \\
\hline ARN 4 & $\begin{array}{l}\text { 4a: } 37.940950,23.648250 \text { - 4b: 37.941363, } 23.648619 \text { - 4c: 37.941887, 23.649127 - 4d: 37.941560, 23.649751 } \\
\text { 4e: 37.941251, 23.650302 - 4f: 37.940781, 23.649910 - 4g: 37.940312, 23.649487 - 4h: 37.940622, 23.648926 }\end{array}$ \\
\hline ARN 5 & $\begin{array}{l}\text { 5a: } 37.942098,23.649270 \text { - 5b: 37.942664, } 23.649770 \text { - 5c: 37.943085, 23.650107 - 5d: } 37.942806,23.650774 \\
\text { 5e: } 37.942424,23.651281 \text { - 5f: 37.941937, } 23.650920 \text { - 5g: 37.941428, 23.650485 - 5h: 37.941747, 23.649841 }\end{array}$ \\
\hline ARN 6 & $\begin{array}{l}\text { 6a: } 37.943197,23.650268 \text { - 6b: 37.943756, } 23.650725 \text { - 6c: 37.944339, 23.651194 - 6d: } 37.944011,23.651859 \\
\text { 6e: } 37.943743,23.652401 \text { - 6f: } 37.943079,23.651867 \text { - 6g: 37.942682, 23.651530 - 6h: } 37.943041,23.650980\end{array}$ \\
\hline ARN 7 & $\begin{array}{l}\text { 7a: } 37.940158,23.649763 \text { - 7b: 37.940619, } 23.650154 \text { - 7c: 37.941137, 23.650589 - 7d: 37.940896, 23.651039 } \\
\text { 7e: } 37.940459,23.651762 \text { - 7f: 37.940014, 23.651017 - 7g: 37.939674, 23.650724 - 7h: 37.939901, 23.650201 }\end{array}$ \\
\hline ARN 8 & $\begin{array}{l}\text { 8a: } 37.941282,23.650730 \text { - 8b: 37.941817, } 23.651155 \text { - 8c: 37.942236, } 23.651555 \text { - 8d: } 37.942084,23.652112 \\
\text { 8e: } 37.941714,23.652786 \text { - 8f: } 37.941344,23.652356 \text { - 8g: 37.940726, 23.651990 - 8h: } 37.941091,23.651150\end{array}$ \\
\hline ARN 9 & $\begin{array}{l}\text { 9a: } 37.942586,23.651776 \text { - 9b: 37.942974, } 23.652134 \text { - 9c: 37.943596, 23.652687 - 9d: } 37.943346,23.653167 \\
\text { 9e: } 37.942959,23.653872 \text { - 9f: } 37.942377,23.653362 \text { - 9g: } 37.941902,23.652866 \text { - 9h: } 37.942278,23.652275\end{array}$ \\
\hline
\end{tabular}


Table 5. The 5QI value and the corresponding constraints for each service.

\begin{tabular}{|c|c|c|c|c|c|}
\hline Service & 5QI Value & Resource Type & Priority Level & $\begin{array}{c}\text { Packet Delay } \\
\text { Budget }\end{array}$ & $\begin{array}{c}\text { Packet Error } \\
\text { Rate }\end{array}$ \\
\hline Autonomous Navigation (ANav) & 81 & Delay Critical GBR & 11 & $5 \mathrm{~ms}$ & $10^{-5}$ \\
\hline Conversational Voice $(\mathrm{CVo})$ & 1 & GBR & 20 & $100 \mathrm{~ms}$ & $10^{-2}$ \\
\hline Conversational Video (CVi) & 2 & GBR & 40 & $150 \mathrm{~ms}$ & $10^{-3}$ \\
\hline Web Browsing (WB) & 6 & Non-GBR & 60 & $300 \mathrm{~ms}$ & $10^{-6}$ \\
\hline
\end{tabular}

Up to 50 vehicles are moving inside the coverage area of each RSU requiring communication resources to satisfy the constraints of their services. In particular, regarding the OSM data about the vehicles' density in the city of Piraeus, Greece, at off-peak hours, the density of vehicles is approximately 10 vehicles per RSU, while at peak hours, the density of vehicles on the map is about 50 vehicles per RSU. At this point, it has to be noted that since the proposed scheme is applied to an urban environment, the average velocity of the vehicles is equal to $9 \mathrm{~m} / \mathrm{s}$, while their moving direction in each road depends on the OSM data about each area of the simulated map. In particular, each vehicle receives one flow for each service. Table 6 presents the simulation parameters.

Table 6. The simulation parameters.

\begin{tabular}{|c|c|}
\hline Parameter & Value \\
\hline Simulation duration & $86,400 \mathrm{~s}(24 \mathrm{~h})$ \\
\hline LTE-V2X RSUs count & 9 LTE-V2X RSUs \\
\hline ARNs count & 1 UAV per RSU \\
\hline Average communication range of each RSU & $100 \mathrm{~m}$ \\
\hline Average communication range of each ARN & $100 \mathrm{~m}$ \\
\hline & Simulation run 1: 10 vehicles \\
\hline & Simulation run 2: 20 vehicles \\
\hline Number of vehicles in the area of each RSU & Simulation run 3: 30 vehicles \\
\hline & Simulation run 4: 40 vehicles \\
\hline & Simulation run 5: 50 vehicles \\
\hline Vehicles' mobility pattern & According to OpenStreetMap (OSM) data \\
\hline ARNs mobility pattern & Stationary \\
\hline Average velocity of vehicles & $9 \mathrm{~m} / \mathrm{s}$ \\
\hline Average height of buildings exist in the map & $30 \mathrm{~m}$ \\
\hline Default altitude of ARNs & $1 \times$ Average_Buildings_Height $=30 \mathrm{~m}$ \\
\hline \multirow{19}{*}{ Services } & Autonomous Navigation (ANav) \\
\hline & - Average datarate per flow: $0.6 \mathrm{Mbps}$ \\
\hline & - Simulated data type: TCP data traffic \\
\hline & - Used NS3 module: NS3 OnOffApplication [59] \\
\hline & Conversational Voice (CVo) \\
\hline & - Average datarate per flow: $200 \mathrm{kbps}$ \\
\hline & - Simulated data type: Voice over IP (VoIP) with G.729 codec \\
\hline & - Used NS3 module: NS3 OnOffApplication [59] \\
\hline & \\
\hline & Conversational Video (CVi) \\
\hline & - Average datarate per flow: $35.3 \mathrm{Mbps}$ (according to Huawei \\
\hline & specifications for $4 \mathrm{~K}$ video with frame rate equal to 60 Frames per \\
\hline & Second (FPS) $[60]$ \\
\hline & - Simulated data type: Live MP4 video \\
\hline & - Used NS3 module: NS3 UDPTraceClient [61] using MP4 video trace \\
\hline & Web Browsing (WB) \\
\hline & - Average datarate per flow: $8.0 \mathrm{Mbps}$ \\
\hline & - Simulated data type: HTTP data traffic \\
\hline & - Used NS3 module: ThreeGppHttpClient [62] \\
\hline
\end{tabular}


The aforementioned simulation setup has been chosen since it implements a realistic scenario for the evaluation of the proposed scheme. In particular, the entire network infrastructure has been built upon a real map, while the vehicles' movement is similar to the movement of real vehicles in the specific geographic area, as it is obtained from the OSM data. In addition, the height of the buildings that exist in the map is configured considering the real height of each building that exists in the simulated geographical area. In general, the average height of the buildings is a critical parameter that affects the performance of the system, since it affects the required altitude of the ARNs as it will be shown in the following paragraphs.

\section{Experimental Results and Discussion}

For the evaluation of the proposed scheme, three different test cases are considered. Specifically, during the first case, only the LTE-V2X RSUs provide communication resources to vehicular users. Accordingly, during the second case, both LTE-V2X RSUs and ARNs are used. In this case, during the instantiation of the system, each ARN obtains a random geographical position inside the coverage area of the corresponding RSU and remains stationary to this position for the entire simulation time. Finally, during the third case, the position of each ARN is optimized by applying the proposed IFT algorithm. In this case, the criteria considered for the evaluation of the alternative positions for each ARN include the vehicles' density in each geographical area, the SINR of the corresponding RSU in this area, as well as the average priorities of the services used from the vehicles moving inside this area. In our case, these criteria are supposed to have equal importance with each other, namely the fact that the corresponding criteria weights are equal to 0.3333 . Furthermore, Table 7 presents the IVIFNs considered for the evaluation of each criterion, while it should be noted that the alternative positions for each ARN are presented in Figure 4.

Table 7. The linguistic terms used for the evaluation of the IFT criteria and the corresponding interval-valued icosagonal fuzzy numbers.

\begin{tabular}{|c|c|c|c|}
\hline $\begin{array}{l}\text { Linguistic Terms Used for the } \\
\text { Vehicles' Density Criterion }\end{array}$ & $\begin{array}{l}\text { Linguistic Terms Used } \\
\text { for the RSU SINR } \\
\text { Criterion }\end{array}$ & $\begin{array}{l}\text { Linguistic Terms Used for the } \\
\text { Vehicles' Services Average } \\
\text { Priorities Criterion }\end{array}$ & $\begin{array}{l}\text { Interval-Valued Icosagonal Fuzzy } \\
\text { Number }\end{array}$ \\
\hline $\begin{array}{l}\text { Very Low Vehicles' Density } \\
\text { (VLVD) }\end{array}$ & Very Low SINR (VLS) & Very Low Service Priority (VLSP) & $\begin{array}{l}{[(0,0,0,0,0,0,0,0,0,0,0.026,0.079,0.132,} \\
0.184,0.237,0.289,0.342,0.395,0.447,0.500, \\
1,0.20,0.40,0.60,0.80,0.80,0.60,0.40,0.20), \\
(0,0,0,0,0,0,0,0,0,0,0.021,0.063,0.105, \\
0.147,0.189,0.232,0.274,0.316,0.358,0.400, \\
0.8,0.08,0.26,0.44,0.62,0.62,0.44,0.26, \\
0.08)]\end{array}$ \\
\hline Low Vehicles' Density (LVD) & Low SINR (LS) & Low Service Priority (LSP) & $\begin{array}{l}{[(0,0,0,0,0,0.013,0.066,0.118,0.171,} \\
0.224,0.276,0.329,0.382,0.434,0.487,0.539, \\
0.592,0.645,0.697,0.750,1,0.20,0.40,0.60, \\
0.80,0.80,0.60,0.40,0.20),(0,0,0,0,0.018, \\
0.061,0.103,0.145,0.187,0.229,0.271,0.313, \\
0.355,0.397,0.439,0.482,0.524,0.566,0.608, \\
0.650,0.8,0.08,0.26,0.44,0.62,0.62,0.44, \\
0.26,0.08)]\end{array}$ \\
\hline
\end{tabular}


Table 7. Cont.

\begin{tabular}{|c|c|c|c|}
\hline $\begin{array}{l}\text { Linguistic Terms Used for the } \\
\text { Vehicles' Density Criterion }\end{array}$ & $\begin{array}{l}\text { Linguistic Terms Used } \\
\text { for the RSU SINR } \\
\text { Criterion }\end{array}$ & $\begin{array}{l}\text { Linguistic Terms Used for the } \\
\text { Vehicles' Services Average } \\
\text { Priorities Criterion }\end{array}$ & $\begin{array}{l}\text { Interval-Valued Icosagonal Fuzzy } \\
\text { Number }\end{array}$ \\
\hline Medium Vehicles' Density (MVD) & Medium SINR (MS) & Medium Service Priority (MSP) & $\begin{array}{l}{[(0,0.053,0.105,0.158,0.211,0.263,0.316,} \\
0.368,0.421,0.474,0.526,0.579,0.632,0.684, \\
0.737,0.789,0.842,0.895,0.947,1,1,0.20, \\
0.40,0.60,0.80,0.80,0.60,0.40,0.20),(0.100, \\
0.142,0.184,0.226,0.268,0.311,0.353,0.395, \\
0.437,0.479,0.521,0.563,0.605,0.647,0.689, \\
0.732,0.774,0.816,0.858,0.900,0.8,0.08, \\
0.26,0.44,0.62,0.62,0.44,0.26,0.08)]\end{array}$ \\
\hline High Vehicles' Density (HVD) & High SINR (HS) & High Service Priority (HSP) & $\begin{array}{l}{[(0.250,0.303,0.355,0.408,0.461,0.513,} \\
0.566,0.618,0.671,0.724,0.776,0.829,0.882, \\
0.934,0.987,1,1,1,1,1,1,0.20,0.40,0.60, \\
0.80,0.80,0.60,0.40,0.20),(0.350,0.392, \\
0.434,0.476,0.518,0.561,0.603,0.645,0.687, \\
0.729,0.771,0.813,0.855,0.897,0.939,0.982, \\
1,1,1,1,0.8,0.08,0.26,0.44,0.62,0.62,0.44, \\
0.26,0.08)]\end{array}$ \\
\hline $\begin{array}{l}\text { Very High Vehicles' Density } \\
\text { (VHVD) }\end{array}$ & Very High SINR (VHS) & Very High Service Priority (VHSP) & $\begin{array}{l}{[(0.500,0.553,0.605,0.658,0.711,0.763,} \\
0.816,0.868,0.921,0.974,1,1,1,1,1,1,1,1, \\
1,1,1,0.20,0.40,0.60,0.80,0.80,0.60,0.40, \\
0.20),(0.600,0.642,0.684,0.726,0.768, \\
0.811,0.853,0.895,0.937,0.979,1,1,1,1,1, \\
1,1,1,1,1,0.8,0.08,0.26,0.44,0.62,0.62, \\
0.44,0.26,0.08)]\end{array}$ \\
\hline
\end{tabular}

Moreover, it should be noted that the IFT algorithm is periodically executed at the SDN controller, for the area of each RSU, in order for the position of the corresponding ARNs to be updated according to the current status of the positions' evaluation criteria. The time interval $T_{r}^{I F T_{\text {execution }}}$ that determines how often the IFT algorithm should be executed for each ARN is estimated using Formula (25), where the $d_{r}$ parameter represents the average distance between the alternative positions that are available for the corresponding $\mathrm{ARN}$, and the $v_{r}$ parameter represents the average velocity of the vehicles in the area of the $r$ RSU which is determined from the OSM data about each specific geographical area. Thus, Table 8 presents the value of the time interval $T_{r}^{I F T_{\text {execution }}}$ parameter obtained for each RSU. Additionally, Table 9 presents the positions that are mostly being selected for each ARN, randomly during the second test case or using the IFT algorithm during the third test case. As it can be observed, in most cases, the IFT algorithm selects a position near a central road where usually the requirements for communication resources are increased:

$$
T_{r}^{I F T_{\text {execution }}}=\frac{d_{r}}{v_{r}}
$$

Table 8. The value of the time interval $T_{r}^{I F T_{\text {execution }}}$ parameter obtained for each RSU.

\begin{tabular}{|c|c|c|c|}
\hline RSU & $\begin{array}{l}\text { Average Distance between the } \\
\text { Alternative Positions }\left(d_{r}\right)\end{array}$ & $\begin{array}{l}\text { Average Velocity of } \\
\text { Vehicles }\left(v_{r}\right)\end{array}$ & $\begin{array}{l}\text { Estimated Time Interval } \\
\qquad\left(T_{r}^{\left.I F T_{\text {execution }}\right)}\right.\end{array}$ \\
\hline 1 & $142.85 \mathrm{~m}$ & $8.33 \mathrm{~m} / \mathrm{s}$ & $17.14 \mathrm{~s}$ \\
\hline 2 & $142.85 \mathrm{~m}$ & $5.55 \mathrm{~m} / \mathrm{s}$ & $25.73 \mathrm{~s}$ \\
\hline 3 & $142.85 \mathrm{~m}$ & $5.55 \mathrm{~m} / \mathrm{s}$ & $25.73 \mathrm{~s}$ \\
\hline 4 & $142.85 \mathrm{~m}$ & $8.33 \mathrm{~m} / \mathrm{s}$ & $17.14 \mathrm{~s}$ \\
\hline 5 & $142.85 \mathrm{~m}$ & $9.72 \mathrm{~m} / \mathrm{s}$ & $14.69 \mathrm{~s}$ \\
\hline 6 & $142.85 \mathrm{~m}$ & $11.11 \mathrm{~m} / \mathrm{s}$ & $12.85 \mathrm{~s}$ \\
\hline 7 & $142.85 \mathrm{~m}$ & $4.16 \mathrm{~m} / \mathrm{s}$ & $34.33 \mathrm{~s}$ \\
\hline 8 & $142.85 \mathrm{~m}$ & $4.16 \mathrm{~m} / \mathrm{s}$ & $34.33 \mathrm{~s}$ \\
\hline 9 & $142.85 \mathrm{~m}$ & $2.77 \mathrm{~m} / \mathrm{s}$ & $51.57 \mathrm{~s}$ \\
\hline
\end{tabular}


Table 9. The ARNs' positions that are mostly being selected in each case.

\begin{tabular}{ccc}
\hline $\begin{array}{c}\text { RSU Serving the } \\
\text { Corresponding Area }\end{array}$ & $\begin{array}{c}\text { Mostly Selected ARN Position for } \\
\text { the Second Case (Randomly) }\end{array}$ & $\begin{array}{c}\text { Mostly Selected ARN Position for } \\
\text { the Third Case (Using the IFT } \\
\text { Algorithm) }\end{array}$ \\
\hline LTE-V2X 1 & $1 \mathrm{~h}$ & $1 \mathrm{~d}$ \\
LTE-V2X 2 & $2 \mathrm{~b}$ & $2 \mathrm{~g}$ \\
LTE-V2X 3 & $3 \mathrm{~b}$ & $3 \mathrm{~d}$ \\
LTE-V2X 4 & $4 \mathrm{~h}$ & $4 \mathrm{e}$ \\
LTE-V2X 5 & $5 \mathrm{c}$ & $5 \mathrm{f}$ \\
LTE-V2X 6 & $6 \mathrm{a}$ & $6 \mathrm{e}$ \\
LTE-V2X 7 & $7 \mathrm{f}$ & $7 \mathrm{c}$ \\
LTE-V2X 8 & $8 \mathrm{~d}$ & $8 \mathrm{c}$ \\
LTE-V2X 9 & $9 \mathrm{~h}$ & $9 \mathrm{~d}$ \\
\hline
\end{tabular}

The parameters considered for the performance evaluation include the throughput, the end-to-end delay, the jitter and the packet loss ratio. As observed from the simulation results, where the positions of the ARNs are optimized, the proposed scheme succeeds improved performance since optimal channel conditions are provided to the vehicular users. In particular, as presented in Figure 5, for the ANav service slice, in the case where ARNs obtain optimized positions, the proposed scheme succeeds up to $2.01 \mathrm{Mbps}$ higher throughput than these succeeded when the ARNs obtain a random position, as well as up to $5.39 \mathrm{Mbps}$ higher throughput in comparison with the case where no ARNs used. At the same time, up to $0.6 \mathrm{~ms}, 0.07 \mathrm{~ms}$ and $17.9 \%$ lower values are observed for both the packet transfer delay, the jitter and the packet loss ratio factors, respectively. Similarly, Figures 6-8 present the corresponding results for the VoIP, the CV and the WB services, respectively. As it can be observed, in the case of the VoIP service slice, where the ARNs obtain optimized positions, up to $0.91 \mathrm{Mbps}$ and $2.45 \mathrm{Mbps}$ higher throughput is achieved, in comparison with the cases where either the ARNs obtain random positions or no ARNs used, respectively. At the same time, for the VoIP service slice, up to $11.27 \mathrm{~ms}, 0.24 \mathrm{~ms}$ and $24.5 \%$ lower values are observed for the delay, the jitter and the packet loss ratio factors, respectively. In addition, similar results are observed in the case of the CV service slice, which is the most demanding service in terms of throughput in our simulation. In particular, in the case of the CV service slice up to $296.7 \mathrm{Mbps}$ and 793.2 Mbps, higher throughput is observed where the positions of the ARNs are optimized, compared with the cases where the ARNs obtain random positions or no ARNs exist. In addition, similar to the other service slices, the values for the delay, the jitter and the packet loss ratio are up to $41.2 \mathrm{~ms}, 12.35 \mathrm{~ms}$ and $44 \%$ lower than those observed in the other two cases, where either ARNs are not used or their geographical positions are not optimized. Finally, for the WB service slice, the proposed scheme succeeds up to $105.5 \mathrm{Mbps}$ and $282.1 \mathrm{Mbps}$ higher throughput from the other two cases, respectively, and up to $157.7 \mathrm{~ms}, 19.71 \mathrm{~ms}$ and $70.5 \%$ lower values for the delay, the jitter and the packet loss ratio, respectively, where the ARNs obtain optimal geographical positions. 


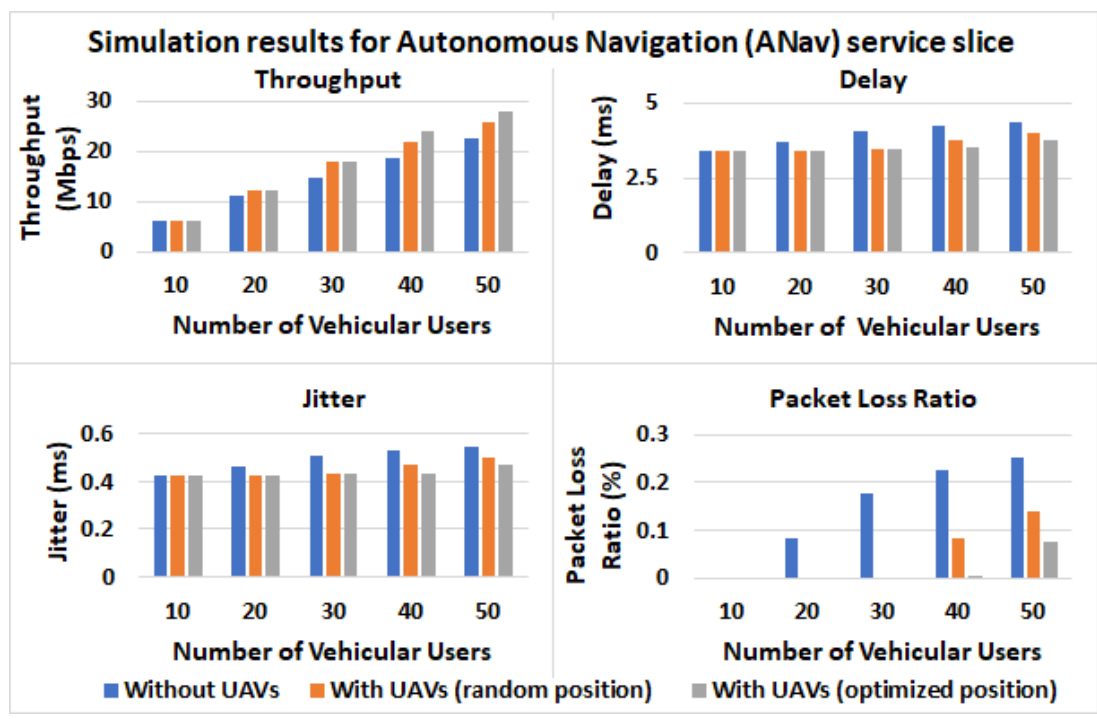

Figure 5. The simulation results for the autonomous navigation (ANav) service slice.

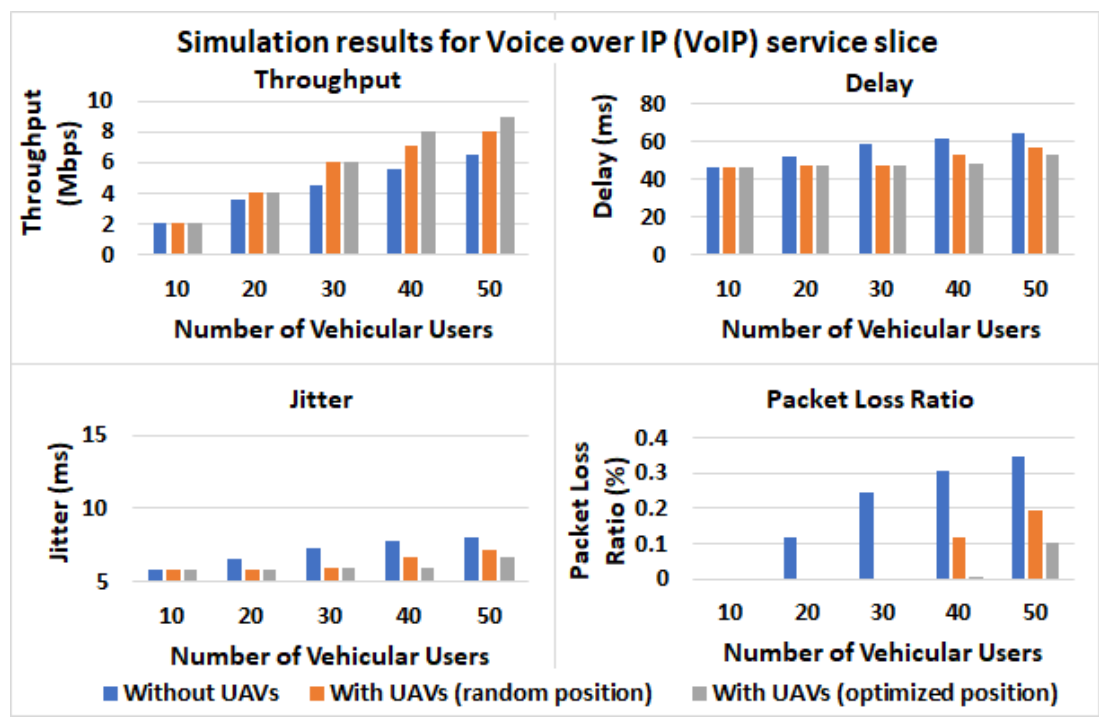

Figure 6. The simulation results for the voice over IP (VoIP) service slice.

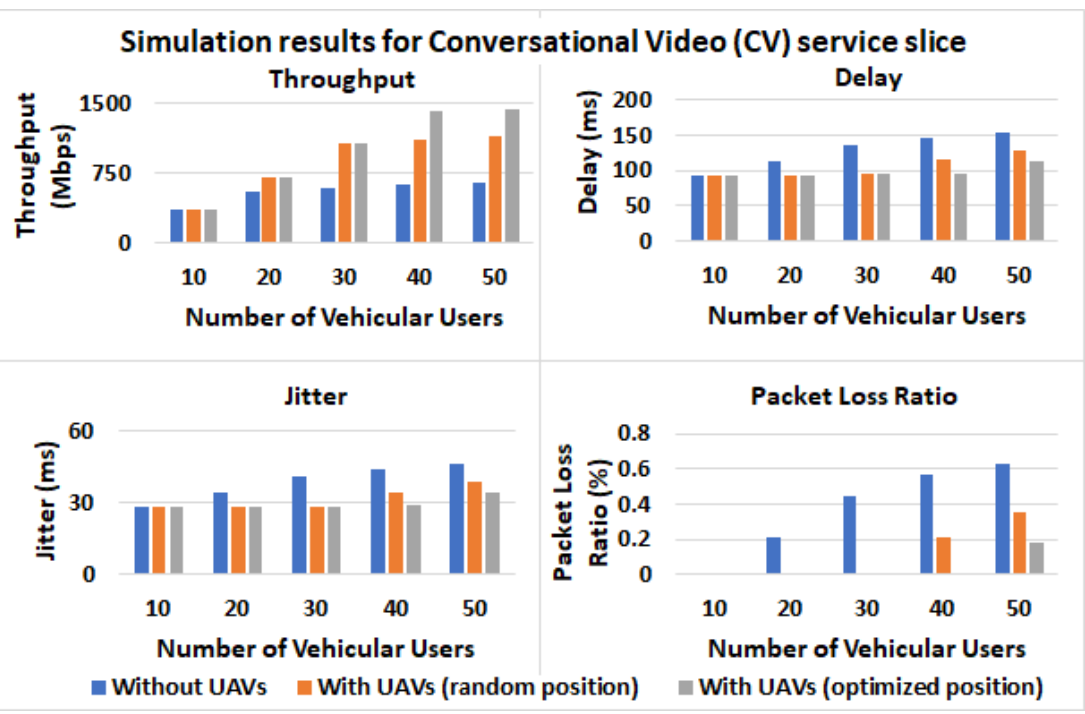

Figure 7. The simulation results for the conversational video $(\mathrm{CV})$ service slice. 


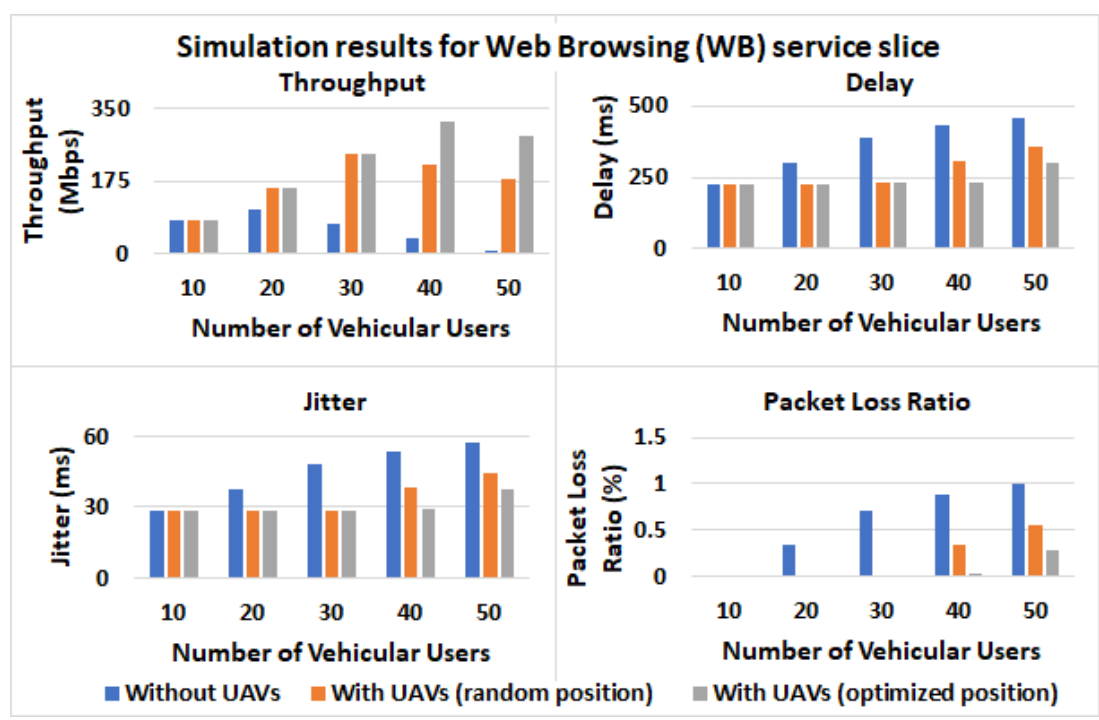

Figure 8. The simulation results for the Web browsing (WB) service slice.

In some cases where the service requirements cannot be met from the RSUs, the use of ARNs results in critical improvements of the system performance and thus to the satisfaction of the constraints of the aforementioned services. Indicatively, in the case of the $\mathrm{CV}$ service, the maximum acceptable is equal to $150 \mathrm{~ms}$. In this case, when the number of vehicular users becomes equal to 50, the observed packet transfer delay is equal to $154.29 \mathrm{~ms}$ (Figure 7) and thus the delay constraint is not satisfied. However, with the use of ARNs, even when their position has not been optimized, the observed packet transfer delay is less than $150 \mathrm{~ms}$ and thus it can be considered a satisfied QoS parameter. Additionally, in the case of the WB service, when the number of vehicular users becomes equal to 50, the delay constraint of the $300 \mathrm{~ms}$ is satisfied only in the case where ARNs with optimized positions are used (Figure 8). In addition, similar improvements are observed in the case of the packet loss ratio factor. Specifically, considering the results for the entire services (Figures 5-8), when no ARNs used the packet loss ratio constraint is satisfied only for the case of 10 users. On the contrary, when ARNs used the packet loss ratio is satisfied for up to 30 users, while in the case where the ARNs obtain optimal positions, the packet loss ratio factor is satisfied for up to 40 users.

Furthermore, Figure 9 presents how the altitude of ARNs affects the performance of the system. As it can be observed, when the altitude of the ARNs is $25 \%$ higher than the average height of the buildings, better performance is observed for the entire services in terms of throughput, delay, jitter and packet loss ratio. On the contrary, worse performance is observed when the ARNs obtain lower altitude than the average height of the building, since in this case, worse channel conditions occur due to the increased number of obstacles that exist between the ARNs and the vehicular users.

Accordingly, Figure 10 presents how the average velocity of the vehicles affects the performance of the system. In particular, as the vehicles move with lower velocity, the performance of the system is maximized since each ARN remains for a longer period of time at a specific position without requiring the execution of the IFT algorithm for selecting another optimal position. On the contrary, when the average velocity of the vehicles is increased, the overall performance of the system is reduced. However, as it is observed, if we compare the results presented in Figure 10 with the ones presented in Figures 6-8, where the vehicles are supposed to have average velocity equal to $9 \mathrm{~m} / \mathrm{s}$, even when the vehicles obtain an average velocity equal to $18 \mathrm{~m} / \mathrm{s}$, the performance achieved from the proposed scheme is better than the one observed in the case where no ARNs used. 


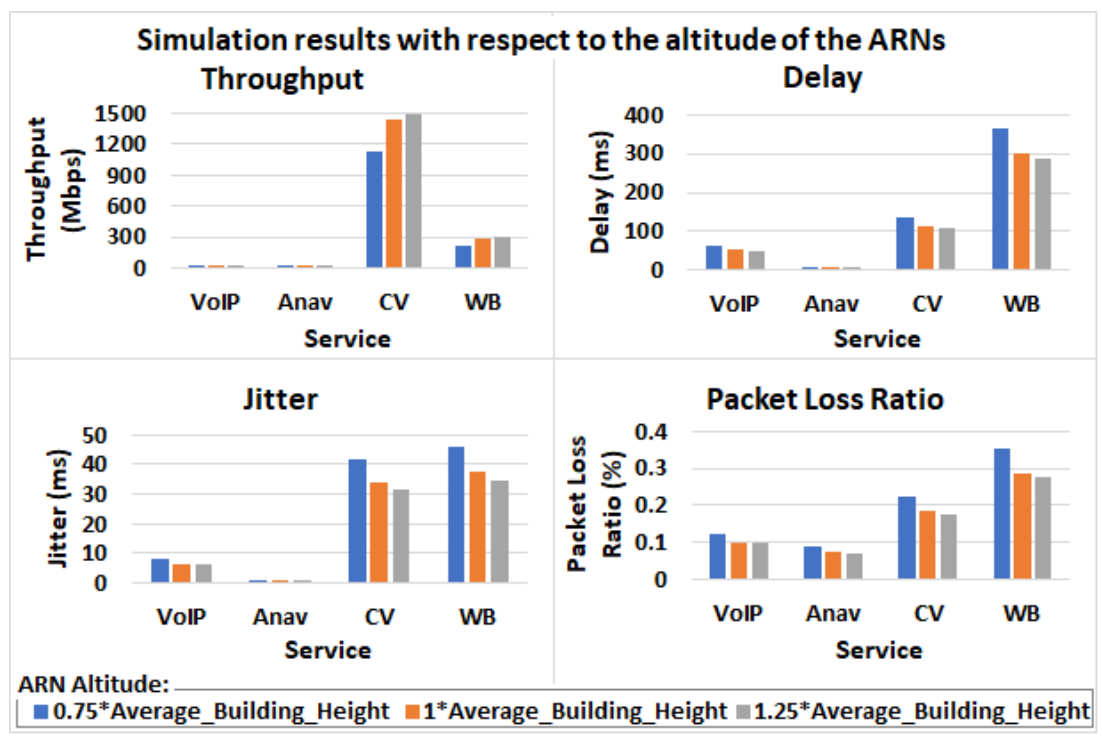

Figure 9. The simulation results with respect to the altitude of the ARNs.

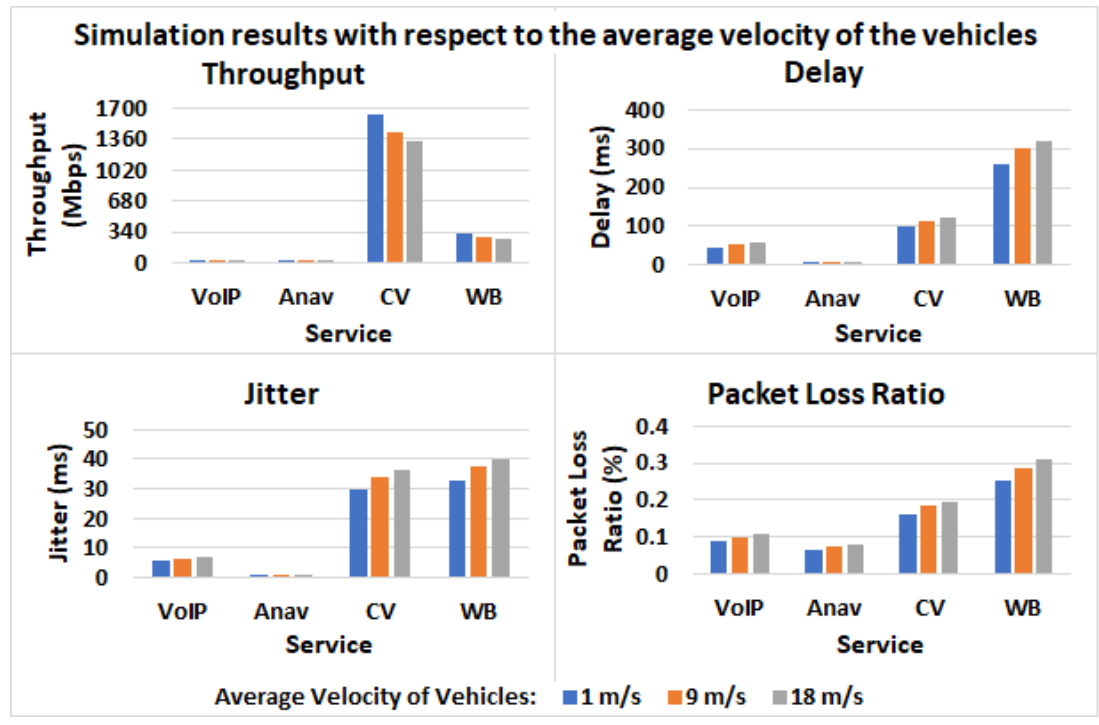

Figure 10. The simulation results with respect to the average velocity of the vehicles.

Regarding the simulation results, it is obvious that when the ARNs obtain optimal positions, even if the same amount of communication resources is committed by the algorithm, improved performance is achieved since improved channel conditions are established. As a conclusion of this situation, it can be said that from the network provider's point of view, this means that communication resources can be released in cases where the requirements of the services are satisfied. On the other hand, from the user's point of view, even better QoS can be perceived.

\section{Conclusions}

In this paper, a network slicing scheme for 5G networks was proposed, aimed at the optimization of the performance of vehicular services. In particular, the proposed network architecture consists of UAVs acting as ARNs and RSUs that provide communication resources to vehicular users. Moreover, the position of each ARN is optimized by applying the proposed IFT algorithm. Regarding the proposed scheme, each ARN maintains its own RBs, while each RSU maintains an LVRP which contains LRBs and SRBs. An SDN controller provides centralized control of the entire architecture, while at the same time, it maintains a VRP, where the SRBs of the RSUs are stored. The satisfaction grade of each user service 
is monitored considering both the QoS and the SINR factors. If the satisfaction grade is higher than the predefined threshold value, the service requirements can be satisfied from the remaining RBs of the RSU or ARN where the user is connected. On the contrary, if the estimated satisfaction grade is lower than the predefined threshold value, the RSU or ARN borrows extra RBs from the corresponding virtual resource pool. Performance evaluation shows that enhanced performance is achieved where the geographical positions of the ARNs are optimized, since in this case, improved channel conditions are provided to the users.

Author Contributions: Conceptualization, E.S., E.T.M. and A.M.; investigation, E.S. and E.T.M.; supervision, A.M. and D.D.V.; visualization, E.S.; writing—original draft, E.S., E.T.M.; writingreview and editing, E.S., E.T.M., A.M., D.J.V., N.I.M. and D.D.V. All authors have read and agreed to the published version of the manuscript.

Funding: This research was partially funded by the University of Western Macedonia Research Committee.

Institutional Review Board Statement: Not applicable since this study does not involve humans or animals.

Informed Consent Statement: Not applicable since this study does not involve humans or animals.

Data Availability Statement: Simulation data can be provided after contacting the corresponding author.

Acknowledgments: This work is partly supported by the University of Western Macedonia Research Committee.

Conflicts of Interest: The authors declare no conflict of interest.

\section{References}

1. Kazemifard, N.; Shah-Mansouri, V. Minimum delay function placement and resource allocation for Open RAN (O-RAN) 5G networks. Comput. Netw. 2021, 188, 107809. [CrossRef]

2. TR 21.915 (V15.0.0): Digital Cellular Telecommunications System (Phase 2+) (GSM); LTE; 5G; (Rel.15); Technical Specification, 3GPP; Universal Mobile Telecommunications System (UMTS): Sophia Antipolis, France, 2019.

3. Jiang, X.; Sheng, M.; Zhao, N.; Xing, C.; Lu, W.; Wang, X. Green UAV communications for 6G: A survey. Chin. J. Aeronaut. 2021. [CrossRef]

4. Raj, A.S.A.; Palanichamy, Y. An aerial intelligent relay-road side unit (AIR-RSU) framework for modern intelligent transportation system. Peer-to-Peer Netw. Appl. 2020, 13, 965-986. [CrossRef]

5. Lemeshko, O.; Yeremenko, O.; Kovalenko, T.; Al-Dulaimi, A.M.; Al-Dulaimi, M.K. Comparative Analysis of the Resource Blocks Allocation Balancing Model in the LTE Downlink Using RAT 1 with Existing Solutions. In Proceedings of the 2018 International Scientific-Practical Conference Problems of Infocommunications, Science and Technology (PIC S\&T), Kharkiv, Ukraine, 9-12 October 2018; pp. 701-704.

6. Raju, V.; Jayagopal, R. An Arithmetic Operations of Icosagonal fuzzy number using Alpha cut. Int. J. Pure Appl. Math. 2018, 120, 137-145.

7. Next Generation Mobile Network Alliance (NGMN). 2021. Available online: https:/ /www.ngmn.org/ (accessed on 20 June 2021).

8. Marinova, S.; Lin, T.; Bannazadeh, H.; Leon-Garcia, A. End-to-end network slicing for future wireless in multi-region cloud platforms. Comput. Netw. 2020, 177, 107298. [CrossRef]

9. Nojima, D.; Katsumata, Y.; Shimojo, T.; Morihiro, Y.; Asai, T.; Yamada, A.; Iwashina, S. Resource isolation in RAN part while utilizing ordinary scheduling algorithm for network slicing. In Proceedings of the 2018 IEEE 87th Vehicular Technology Conference (VTC Spring), Porto, Portugal, 3-6 June 2018; pp. 1-5.

10. Joung, J. Random space-time line code with proportional fairness scheduling. IEEE Access 2020, 8, 35253-35262. [CrossRef]

11. Ge, X. Ultra-reliable low-latency communications in autonomous vehicular networks. IEEE Trans. Veh. Technol. 2019, 68, 5005-5016. [CrossRef]

12. Piro, G.; Grieco, L.A.; Boggia, G.; Fortuna, R.; Camarda, P. Two-level downlink scheduling for real-time multimedia services in LTE networks. IEEE Trans. Multimed. 2011, 13, 1052-1065. [CrossRef]

13. Ouaissa, M.; Rhattoy, A.; Lahmer, M. Comparative performance study of QoS downlink scheduling algorithms in LTE system for M2M communications. In Proceedings of the International Conference Europe Middle East \& North Africa Information Systems and Technologies to Support Learning, Fez, Morocco, 25-27 October 2018; Springer: Berlin/Heidelberg, Germany, 2018; pp. 216-224.

14. Skondras, E.; Michalas, A.; Sgora, A.; Vergados, D.D. A downlink scheduler supporting real time services in LTE cellular networks. In Proceedings of the 2015 6th International Conference on Information, Intelligence, Systems and Applications (IISA), Corfu, Greece, 6-8 July 2015; pp. 1-6. 
15. Skondras, E.; Michalas, A.; Sgora, A.; Vergados, D.D. QoS-aware scheduling in LTE-A networks with SDN control. In Proceedings of the 2016 7th International Conference on Information, Intelligence, Systems \& Applications (IISA), Chalkidiki, Greece, 13-15 July 2016; pp. 1-6.

16. Mihovska, A.; Prasad, R. Overview of 5G New Radio and Carrier Aggregation: 5G and Beyond Networks. In Proceedings of the 2020 23rd International Symposium on Wireless Personal Multimedia Communications (WPMC), Okayama, Japan, 18-26 October 2020; pp. 1-6.

17. Haci, H.; Abdelbari, A. Throughput enhanced scheduling (TES) scheme for ultra-dense networks. Int. J. Commun. Syst. 2020, 33, e4229. [CrossRef]

18. Shi, W.; Zhou, H.; Li, J.; Xu, W.; Zhang, N.; Shen, X. Drone assisted vehicular networks: Architecture, challenges and opportunities. IEEE Netw. 2018, 32, 130-137. [CrossRef]

19. Michailidis, E.T.; Potirakis, S.M.; Kanatas, A.G. AI-Inspired Non-Terrestrial Networks for IIoT: Review on Enabling Technologies and Applications. IoT 2020, 1, 21-48. [CrossRef]

20. Khabbaz, M.; Antoun, J.; Assi, C. Modeling and performance analysis of UAV-assisted vehicular networks. IEEE Trans. Veh. Technol. 2019, 68, 8384-8396. [CrossRef]

21. Fawaz, W. Effect of non-cooperative vehicles on path connectivity in vehicular networks: A theoretical analysis and UAV-based remedy. Veh. Commun. 2018, 11, 12-19. [CrossRef]

22. Zhou, Y.; Cheng, N.; Lu, N.; Shen, X.S. Multi-UAV-aided networks: Aerial-ground cooperative vehicular networking architecture. IEEE Veh. Technol. Mag. 2015, 10, 36-44. [CrossRef]

23. Hong, K.; Lillethun, D.; Ramachandran, U.; Ottenwälder, B.; Koldehofe, B. Mobile fog: A programming model for large-scale applications on the internet of things. In Proceedings of the Second ACM SIGCOMM Workshop on Mobile Cloud Computing, Hong Kong, China, 16 August 2013; pp. 15-20.

24. Bithas, P.S.; Michailidis, E.T.; Nomikos, N.; Vouyioukas, D.; Kanatas, A.G. A survey on machine-learning techniques for UAV-based communications. Sensors 2019, 19, 5170. [CrossRef] [PubMed]

25. Secinti, G.; Darian, P.B.; Canberk, B.; Chowdhury, K.R. SDNs in the sky: Robust end-to-end connectivity for aerial vehicular networks. IEEE Commun. Mag. 2018, 56, 16-21. [CrossRef]

26. Nomikos, N.; Michailidis, E.T.; Trakadas, P.; Vouyioukas, D.; Karl, H.; Martrat, J.; Zahariadis, T.; Papadopoulos, K.; Voliotis, S. A UAV-based moving 5G RAN for massive connectivity of mobile users and IoT devices. Veh. Commun. 2020, 25, 100250. [CrossRef]

27. Michailidis, E.T.; Miridakis, N.I.; Michalas, A.; Skondras, E.; Vergados, D.J. Energy Optimization in Dual-RIS UAV-Aided MEC-Enabled Internet of Vehicles. Sensors 2021, 21, 4392. [CrossRef]

28. Cheng, N.; Xu, W.; Shi, W.; Zhou, Y.; Lu, N.; Zhou, H.; Shen, X. Air-ground integrated mobile edge networks: Architecture, challenges, and opportunities. IEEE Commun. Mag. 2018, 56, 26-32. [CrossRef]

29. Faraci, G.; Grasso, C.; Schembra, G. Design of a 5G network slice extension with MEC UAVs managed with reinforcement learning. IEEE J. Sel. Areas Commun. 2020, 38, 2356-2371. [CrossRef]

30. Yuan, Z.; Muntean, G.M. AirSlice: A Network Slicing Framework for UAV Communications. IEEE Commun. Mag. 2020, 58, 62-68. [CrossRef]

31. Xilouris, G.K.; Batistatos, M.C.; Athanasiadou, G.E.; Tsoulos, G.; Pervaiz, H.B.; Zarakovitis, C.C. UAV-assisted 5G network architecture with slicing and virtualization. In Proceedings of the 2018 IEEE Globecom Workshops (GC Wkshps), Abu Dhabi, United Arab Emirates, 9-13 December 2018; pp. 1-7.

32. Garcia, A.E.; Hofmann, S.; Sous, C.; Garcia, L.; Baltaci, A.; Bach, C.; Wellens, R.; Gera, D.; Schupke, D.; Gonzalez, H.E. Performance evaluation of network slicing for aerial vehicle communications. In Proceedings of the 2019 IEEE International Conference on Communications Workshops (ICC Workshops), Shanghai, China, 22-24 May 2019; pp. 1-6.

33. Zhang, S.; Quan, W.; Li, J.; Shi, W.; Yang, P.; Shen, X. Air-ground integrated vehicular network slicing with content pushing and caching. IEEE J. Sel. Areas Commun. 2018, 36, 2114-2127. [CrossRef]

34. Lyu, F.; Yang, P.; Wu, H.; Zhou, C.; Ren, J.; Zhang, Y.; Shen, X. Service-Oriented Dynamic Resource Slicing and Optimization for Space-Air-Ground Integrated Vehicular Networks. IEEE Trans. Intell. Transp. Syst. 2021. [CrossRef]

35. Grasso, C.; Schembra, G. A fleet of MEC UAVs to extend a 5 G network slice for video monitoring with low-latency constraints. J. Sens. Actuator Netw. 2019, 8, 3. [CrossRef]

36. Skondras, E.; Michalas, A.; Vergados, D.J.; Michailidis, E.T.; Miridakis, N.I.; Vergados, D.D. Network Slicing on 5G Vehicular Cloud Computing Systems. Electronics 2021, 10, 1474. [CrossRef]

37. Zadeh, L.A. Fuzzy logic. Computer 1988, 21, 83-93. [CrossRef]

38. Singh, H.; Gupta, M.M.; Meitzler, T.; Hou, Z.G.; Garg, K.K.; Solo, A.M.; Zadeh, L.A. Real-life applications of fuzzy logic. Adv. Fuzzy Syst. 2013. [CrossRef]

39. View on 5G Architecture (Version 3); 5G PPP Architecture Working Group: Heidelberg, Germany, 2019.

40. Skondras, E.; Michalas, A.; Vergados, D.D. Mobility management on 5 g vehicular cloud computing systems. Veh. Commun. 2019, 16, 15-44. [CrossRef]

41. Desogus, C.; Anedda, M.; Murroni, M. Real-time load optimization for multimedia delivery content over heterogeneuos wireless network using a MEW approach. In Proceedings of the 2017 IEEE International Symposium on Broadband Multimedia Systems and Broadcasting (BMSB), Cagliari, Italy, 7-9 June 2017; pp. 1-4. 
42. Skondras, E.; Michalas, A.; Vergados, D.J.; Michailidis, E.T.; Miridakis, N.I. A Network Slicing Algorithm for 5G Vehicular Networks. In Proceedings of the 2021 12th International Conference on Information, Intelligence, Systems and Applications (IISA), Crete, Greece, 12-14 July 2021; pp. 1-6.

43. Skondras, E.; Sgora, A.; Michalas, A.; Vergados, D.D. An analytic network process and trapezoidal interval-valued fuzzy technique for order preference by similarity to ideal solution network access selection method. Int. J. Commun. Syst. 2016, 29, 307-329. [CrossRef]

44. Charilas, D.E.; Markaki, O.I.; Psarras, J.; Constantinou, P. Application of fuzzy AHP and ELECTRE to network selection. In Mobile Lightweight Wireless Systems; Springer: Berlin/Heidelberg, Germany, 2009; pp. 63-73.

45. Goyal, R.K.; Kaushal, S.; Sangaiah, A.K. The utility based non-linear fuzzy AHP optimization model for network selection in heterogeneous wireless networks. Appl. Soft Comput. 2017, 67, 800-811. [CrossRef]

46. Skondras, E.; Michalas, A.; Tsolis, N.; Vergados, D.D. A network selection scheme with adaptive criteria weights for $5 \mathrm{~g}$ vehicular systems. In Proceedings of the 2018 9th International Conference on Information, Intelligence, Systems and Applications (IISA), Zakynthos, Greece, 23-25 July 2018; pp. 1-7.

47. Skondras, E.; Michalas, A.; Tsolis, N.; Vergados, D.D. A VHO scheme for supporting healthcare services in 5G vehicular cloud computing systems. In Proceedings of the 2018 Wireless Telecommunications Symposium (WTS), Phoenix, AZ, USA, 17-20 April 2018; pp. 1-6.

48. Skondras, E.; Siountri, K.; Michalas, A.; Vergados, D.D. A route selection scheme for supporting virtual tours in sites with cultural interest using drones. In Proceedings of the 2018 9th International Conference on Information, Intelligence, Systems and Applications (IISA), Zakynthos, Greece, 23-25 July 2018; pp. 1-6.

49. Hwang, C.L.; Yoon, K. Multiple Attribute Decision Making; Springer: Berlin/Heidelberg, Germany, 1981.

50. Ncibi, K.; Hadji, R.; Hamdi, M.; Mokadem, N.; Abbes, M.; Khelifi, F.; Zighmi, K.; Hamed, Y. Application of the analytic hierarchy process to weight the criteria used to determine the Water Quality Index of groundwater in the northeastern basin of the Sidi Bouzid region, Central Tunisia. Euro-Mediterr. J. Environ. Integr. 2020, 5, 1-15. [CrossRef]

51. Elshaboury, N.; Attia, T.; Marzouk, M. Comparison of several aggregation techniques for deriving analytic network process weights. Water Resour. Manag. 2020, 34, 4901-4919. [CrossRef]

52. Ashtiani, B.; Haghighirad, F.; Makui, A. Extension of fuzzy TOPSIS method based on interval-valued fuzzy sets. Appl. Soft Comput. 2009, 9, 457-461. [CrossRef]

53. Open Street Map (OSM). Available online: https:/ / www.openstreetmap.org (accessed on 20 June 2021).

54. Behrisch, M.; Bieker, L.; Erdmann, J.; Krajzewicz, D. SUMO-simulation of urban mobility: An overview. In Proceedings of the SIMUL 2011, The Third International Conference on Advances in System Simulation, Barcelona, Spain, $23-29$ October 2011.

55. Network Simulator 3 (NS3). Available online: https:/ / www.nsnam.org/ (accessed on 20 June 2021).

56. NS3 LTE-V2X Extension Module. Available online: https:/ / github.com/ eisbaer/v2x-lte (accessed on 20 June 2021).

57. TR 36.814 (V9.0.0): Further advancements for E-UTRA physical layer aspects (Release 9). In Technical Specification Group Radio Access Network, 3GPP; Universal Mobile Telecommunications System (UMTS): Sophia Antipolis, France, 2010.

58. TR 36.942 (V10.2.0): Radio Frequency (RF) system scenarios (Release 10). In Technical Specification Group Radio Access Network, 3GPP; Universal Mobile Telecommunications System (UMTS): Sophia Antipolis, France, 2011.

59. NS3 OnOffApplication Class Reference. Available online: https://www.nsnam.org/doxygen/classns3_1_1_on_off_application. html (accessed on 20 June 2021).

60. iLab Video Gene Big Data Team. Video Big Data: The Top 10 Most Demanding Videos on the Net. In Video Big Data Report; iLab Video Gene Big Data Team: Shenzhen, China, 2017,

61. NS3 UdpTraceClient Class Reference. 2021. Available online: https://www.nsnam.org/doxygen/structns3_1_1_udp_trace_ client.html (accessed on 20 June 2021).

62. 3GPP HTTP applications, NS3. 2021. Available online: https://www.nsnam.org/docs/models/html/applications.html (accessed on 20 June 2021). 